\title{
Ubiquitin-Dependent Rapid Degradation Conceals A Cell-Protective Function of Cytoplasmic SIRT3 Against Oxidative Stress
}

\section{Takashi Hayashi}

Sapporo Medical University

Takashi Matsushita

Sapporo Medical University

\section{Shin Hisahara}

Sapporo Medical University

Naotoshi Iwahara

Sapporo Medical University

\section{Atsushi Kuno}

Sapporo Medical University

\section{Risa Kunimoto}

Sapporo Medical University

\section{Ryusuke Hosoda}

Sapporo Medical University

Masaya Tanno

Sapporo Medical University

Shun Shimohama

Sapporo Medical University

Yoshiyuki Horio ( $\square$ horio@sapmed.ac.jp )

Sapporo Medical University

\section{Research Article}

Keywords: SIRT3, COS7, FLAG, nuclear fraction

Posted Date: December 3rd, 2020

DOI: https://doi.org/10.21203/rs.3.rs-112500/v1

License: (1) (1) This work is licensed under a Creative Commons Attribution 4.0 International License.

Read Full License 
Version of Record: A version of this preprint was published at The Journal of Biochemistry on October 29th, 2021. See the published version at https://doi.org/10.1093/jb/mvab119. 


\section{Ubiquitin-dependent rapid degradation conceals a cell-protective function of}

\section{cytoplasmic SIRT3 against oxidative stress}

Takashi Hayashi $^{* 1,2}$, Takashi Matsushita ${ }^{* 1,2}$, Shin Hisahara ${ }^{2}$, Naotoshi Iwahara ${ }^{1,2}$,

Atsushi Kuno ${ }^{1}$, Risa Kunimoto ${ }^{1}$, Ryusuke Hosoda ${ }^{1}$, Masaya Tanno ${ }^{3}$, Shun Shimohama ${ }^{2}$, Yoshiyuki Horio $^{1 * *}$

Departments of ${ }^{1}$ Pharmacology, ${ }^{2}$ Neurology and ${ }^{3}$ Cardiovascular, Renal and Metabolic Medicine, Sapporo Medical University School of Medicine, Sapporo 060-8556, Japan.

*Equal contribution to this study.

**Address for correspondence

Yoshiyuki Horio

Department of Pharmacology, Sapporo Medical University School of Medicine, South-

1, West-17, Chuo-ku Sapporo 060-8556, Japan

Phone: 81-11-611-2111 (Ext. 27200)

Fax: 81-11-612-5860

E-mail: horio@sapmed.ac.jp

\section{Abstract}


SIRT3 is a NAD ${ }^{+}$-dependent protein deacetylase localized in mitochondria. Although several previous studies reported cytoplasmic and/or nuclear localization of SIRT3, extra-mitochondrial SIRT3 was obscure. We found that mitochondrial (SIRT3mt) and cytoplasmic (SIRT3ct) Sirt3 mRNAs were expressed in the mouse brain and diffuse SIRT3 immunostaining in cytoplasm was detected in cultured neural cells and neural precursor cells where SIRT3 knockdown disturbed neural precursor cell differentiation. However, overexpression of SIRT3 in COS7 cells showed that expression levels of SIRT3ct was much lower than that of SIRT3mt. SIRT3ct but not SIRT3mt was promptly degraded by the ubiquitin-dependent degradation, in which SIRT3ct degradation was mediated mainly by the ubiquitination of $\mathrm{NH}_{2}$-terminal methionine and partly by that of lysine residues. SIRT3ct expression level was significantly enhanced by the treatment of cells with staurosporine or $\mathrm{H}_{2} \mathrm{O}_{2} . \mathrm{H}_{2} \mathrm{O}_{2}$ promoted nuclear translocation of SIRT3ct and induced histone H3 deacetylation and superoxide dismutase 2 expression. The overexpression of SIRT3ct decreased cell death by $\mathrm{H}_{2} \mathrm{O}_{2}$ at similar levels achieved by that of SIRT3mt. Knockdown of Sirt3 mRNA increased cell death by amyloid- $\beta(A \beta)$ and the overexpression of SIRT3ct opposed the toxic function of $\mathrm{A} \beta$ in PC12 cells. These results indicated that SIRT3ct participated in cell survival under various stress conditions. 


\section{Introduction}

SIRT3, a member of the sirtuin family, deacetylates acetylated proteins with NAD ${ }^{+}$ resulting in deacetylated proteins, O-acetyl-ADP-ribose and nicotinamide ${ }^{1}$. Among the proteins of the sirtuin family, SIRT1 and SIRT2 localize both in the cytoplasm and nucleus. SIRT3, SIRT4 and SIRT5 are mitochondrial proteins, whereas SIRT6 and SIRT7 are nuclear proteins ${ }^{1}$. The human SIRT3 protein contains a mitochondrial targeting sequence in its $\mathrm{NH}_{2}$-terminal region and is localized in the mitochondrial matrix $^{2}$. SIRT3 regulates various mitochondrial proteins and participates in stress resistance, oxidative phosphorylation, lipolysis and urea formation. SIRT3 deacetylates and activates superoxide dismutase 2 (SOD2) and isocitrate dehydrogenase 2 in mitochondria, resulting in a decrease of superoxide anion and an increase in glutathione levels $^{3,4}$. SIRT3 has been shown to ameliorate various pathological conditions. In the heart, SIRT3 has a protective role in cardiac ischemia, hypertrophy and fibrosis ${ }^{5}$. In the brain, SIRT3 is involved in the adaptive antioxidant response and prevents neuronal death driven by wakefulness in locus ceruleus ${ }^{6}$ and in the mouse model of epilepsy and Huntington's disease ${ }^{7}$. Although most studies show that SIRT3 is exclusively localized in mitochondria, several reports have argued cytoplasmic and/or nuclear localization of SIRT3 $^{8-13}$. Scher et al. showed that two isoforms of SIRT3 existed and full length and 
processed forms of SIRT3 localized to the nucleus and mitochondria, respectively ${ }^{8}$. In the nucleus, SIRT3 could deacetylate histones and was recruited into the mitochondria upon cell stress ${ }^{8}$. Nakamura et al. reported that co-expression of SIRT5 recruited SIRT3 from mitochondria to the nucleus ${ }^{9}$. Cooper et al. showed that equal expression levels of mitochondrial and extra-mitochondrial SIRT3 subtypes were expressed in mouse NIH3T3 cells ${ }^{10}$. Bao et al. reported that a long isoform preferably localized in mitochondria compared with that of the short isoform ${ }^{11}$. Iwahara et al. reported that nuclear SIRT3 was subjected to rapid degradation under stress conditions ${ }^{12}$. Weir et al. reported that SIRT3 localized in the mitochondria, nucleus and cytoplasm in the brain ${ }^{13}$. Thus, there is no doubt about the localization and functions of mitochondrial SIRT3, but that of cytoplasmic or nuclear SIRT3 are still obscure.

In this study, we found that Sirt3 mRNA for cytoplasmic SIRT3 (SIRT3ct) was highly expressed in the brain but the SIRT3ct protein level was much lower than that of SIRT3mt. We found that SIRT3ct was rapidly degraded by a unique ubiquitindependent pathway, which might be regulated by cellular stress. SIRT3ct promoted cell survival against oxidative stress and amyloid- $\beta(\mathrm{A} \beta)$. Our study suggested a latent and important role of SIRT3ct, which became manifest under stress conditions in the brain.

\section{Results}




\section{Comparison of mitochondrial and cytoplasmic SIRT3.}

Five types of mouse Sirt3 $m R N A s$ have been reported in the NCBI Reference Sequence Database and three forms, Sirt3mt, Sirt3ct1 and Sirt3ct2, are assumed to encode active enzymes as shown in Fig. 1a. These Sirt3 mRNAs are generated by alternative splicing of the mouse SIRT3 gene. We screened $6 \times 10^{5}$ phage clones of a mouse brain cDNA library and obtained seventeen SIRT3 cDNAs. Among them, two clones were cDNAs for Sirt3mt (NM_001177804.1) that encoded the SIRT3mt protein of 334 amino acids with a putative mitochondrial targeting sequence (Fig. 1a-c). The remaining fifteen cDNA clones were identical to Sirt3ct1 (NM_001127351.1) which encoded SIRT3ct protein of 257 amino acids without a mitochondrial targeting sequence (Fig. 1a,c). We could not detect a cDNA clone for Sirt3ct2 (NM_022433.2) in the brain. The NCBI reference sequences show that human SIRT3 proteins consist of two isoforms depicted as human SIRT3mt and human SIRT3ct (Fig. 1c). The $\mathrm{NH}_{2}$-terminal sequence corresponding to residues 1-25 of human SIRT3mt is required for mitochondrial import of the protein ${ }^{2}$. Amino acid residues $15-35$ of mouse SIRT3mt had high similarity to the $\mathrm{NH}_{2}$-terminal mitochondrial targeting sequence of human SIRT3mt (Fig. 1b,c) and contained a cluster of charged arginine residues opposed by a hydrophobic cluster in a helical wheel plot (right side of Fig. 1b), suggesting that this region had an amphipathic- 
$\alpha$-helix, a feature of mitochondrial targeting sequences ${ }^{2}$. During translocation of mitochondrial proteins from cytoplasm to mitochondria, mitochondrial targeting sequences are cleaved by mitochondrial processing peptidase (MPP). MPP cuts mitochondrial targeting sequences which contain arginine residues at positions -10 and 2 and/or -3 from the cleavage site ${ }^{14}$. The targeting sequences also have a proline residue between the distal and proximal arginine residues ${ }^{14}$. Arginine residues at positions 84 , 99, and 100, and proline residue at 88 of human SIRT3mt are consistent with the consensus amino acid sequence of mitochondrial targeting sequences and arginine residues at 99 and 100 of human SIRT3mt are shown to be necessary for the cleavage by MPP, which produces $28 \mathrm{kDa}$ human SIRT3mt in mitochondria ${ }^{2}$. Mouse SIRT3mt had arginine residues at positions of 27, 35, and 36 and a proline residue at position 31 , which matched with consensus mitochondrial targeting sequences, suggesting that the amino acid sequence of 1-37 was the mitochondrial targeting sequence of mouse SIRT3mt (Fig. 1b,c).

To examine the subcellular localization of mouse SIRT3mt and SIRT3ct, SIRT3mt and SIRT3ct were tagged with EGFP in their $\mathrm{COOH}$-terminal regions and expressed in COS7 cells, in which mitochondria were stained with MitoTracker Red. SIRT3mt-EGFP mainly localized in the mitochondria, whereas SIRT3ct-EGFP was 
found in the cytoplasm (Fig. 1d). SIRT3mt-EGFP was also found in the extra-

mitochondrial cytoplasmic region where SIRT3mt-EGFP showed a dot-like appearance (Fig. 1d). Interestingly, SIRT3ct-EGFP frequently showed a dot-like appearance in the cytoplasm (Fig. 1d). To further investigate the localization of SIRT3mt and SIRT3ct, the two isoforms were fused with a FLAG-tag at $\mathrm{COOH}$-terminal ends and expressed in COS7 cells, and cells were then homogenized and fractionated into nuclear $(\mathrm{Nu})$, cytoplasmic $(\mathrm{Ct})$ and mitochondrial fractions $(\mathrm{Mt})$. Lamin A/C, cytoplasmic aspartate aminotransferase (cAST) and mitochondrial aspartate aminotransferase (mAST) are nuclear, cytoplasmic and mitochondrial marker proteins, respectively. Immunoblot analysis showed that expression of SIRT3mt-FLAG cDNA gave rise to three bands of SIRT3mt-FLAG proteins $(37,33$, and $32 \mathrm{kDa})$, which were dominantly detected in the mitochondrial fraction and also in the cytoplasmic fraction (Fig. 1e). The intensity of the band of the smallest SIRT3mt (32 kDa) was higher in the mitochondrial fraction than that of the cytoplasmic fraction (Fig. 1e). A small amount of $32 \mathrm{kDa}$ SIRT3mtFLAG was detected in the nuclear fraction (Fig. 1e). SIRT3ct-FLAG was present in the cytoplasmic fraction and its molecular weight was estimated to be $28 \mathrm{kDa}$ (Fig. 1e). A small amount of SIRT3ct-FLAG was also detected in the nuclear and mitochondrial fractions (Fig. 1e). Although the same DNA amounts of SIRT3ct-FLAG and SIRT3mt- 
FLAG cDNAs were transfected in COS7 cells, the intensity of SIRT3ct-FLAG bands in immunoblotting was always much weaker than those of SIRT3mt-FLAG (Fig. 1e).

SIRT3 is involved in stress resistance of cells ${ }^{1,5}$. We examined the effect of staurosporine (SP), a non-genotoxic stressor, on the expression and distribution of SIRT3mt-FLAG and SIRT3ct-FLAG. Homogenates of COS7 cells transfected with the same amount of cDNA for SIRT3mt-FLAG or SIRT3ct-FLAG were fractionated into the nuclear fraction $(\mathrm{Nu})$ and the extra-nuclear fraction (En) containing cytoplasm and mitochondria (Fig. 1f). SP treatment enhanced the expression levels of SIRT3mt-FLAG proteins of $37 \mathrm{kDa}$ and $33 \mathrm{kDa}$ in the nuclear and extra-nuclear fractions, whereas the intensity of the smallest SIRT3mt-FLAG of $32 \mathrm{kDa}$ was not affected by SP treatment (Fig. 1f). SP treatment significantly increased the SIRT3ct-FLAG expression level in the cytoplasm (Fig. 1f). A small amount of SIRT3ct-FLAG band was also detected in the nuclear fraction after SP treatment (Fig. 1f).

\section{Expression of SIRT3 in the brain and neural precursor cells.}

We examined the expression of Sirt3 $m R N A$ in various organs. Northern blot analysis showed that Sirt3 mRNA was highly expressed in the heart, brain, kidney and embryo (Fig. 2a) and was constantly expressed in the mouse brain of various developmental stages and ages (Fig. 2b). Developmental change of the expression of Sirt3 mRNA in the 
fetuses showed that Sirt3 mRNA levels were high in the embryonic day (E) 4.5 to E6.5 and then reduced. In the late embryonic stages, Sirt3 mRNA levels increased again and the highest expression levels were found at E16.5-E18.5 (Fig. 2c). RT-PCR experiments using the fetal and post-natal brain also showed higher expression levels of Sirt3 $m R N A$ at E14.5 and P2 compared with that of E10.5 (Fig. 2d). Immunostaining of SIRT3 in the mouse brain showed that high levels of SIRT3 expression were found in the fetal brain and P2 mouse brain where cells around cerebral ventricles expressed high levels of SIRT3 (Fig. 2e).

SIRT1, another $\mathrm{NAD}^{+}$-dependent protein deacetylase, is highly expressed in neural precursor cells (NPCs) and participates in neuronal differentiation ${ }^{15}$. To examine whether NPCs express SIRT3, neurospheres were cultured from the striatum of the E14.5 mouse brain and were immunostained with an anti-SIRT3 antibody. Similar to SIRT1, SIRT3 was highly expressed in NPCs and almost all of the SIRT3 ${ }^{+}$cells expressed nestin, a marker of NPCs (Fig. 2f). Costaining of SIRT3 with nestin indicated that SIRT3 was diffusely expressed in the cytoplasm but not in the nucleus.

SIRT3 protein expression was also examined in the adult mouse brain. Immunostaining showed that SIRT3 was highly expressed in some subsets of neural cells in such as the hippocampus, cerebral cortex, cerebellum and in the ependyma (Fig. 
$2 \mathrm{~g}$ ). To confirm the expression of SIRT3 in neurons, cortical neurons were isolated from newborn mouse brains and cultured. The cells were immunostained with an antiSIRT3 antibody and an anti- $\beta$ III-tubulin antibody, a marker of neural cells.

Mitochondria were stained with MitoTracker Red. SIRT3 distributed diffusely in cortical neurons, in which SIRT3 was detected not only in the mitochondria but also in the cytoplasm (Fig. 2h). Cellular protrusions were also stained by the SIRT3 antibody (Fig. 2h).

\section{Knockdown of SIRT3 disturbed differentiation of neural precursor cells.}

SIRT3 is highly expressed in the subventricular zone of the brain and in cultured NPCs

(Fig. 2e,f). To examine the distribution of SIRT3 in NPCs, we dissociated NPCs of neurospheres and immunostained them with antibodies against SIRT3 and cytochrome C, a marker of mitochondria. Diffuse cytoplasmic immunostaining of SIRT3 suggested that SIRT3 protein localized in both the cytoplasm and mitochondria (Fig. 3a). In the proliferation condition, neurospheres were round and highly expressed SIRT3 and SIRT1 (Fig. 3b). Expression of $\beta$ III-tubulin, a marker of neuronal cells, was not detected (Fig. 3b). Neurospheres can differentiate into neurons, oligodendrocytes and astrocytes under differentiation condition. When neurospheres were cultured in the differentiation medium for 5 days, they became flat and differentiated cells were 
scattered around spheres. Under differentiation condition, expression of SIRT3 and SIRT1 was suppressed and $\beta$ III-tubulin ${ }^{+}$cells appeared (Fig. 3b), suggesting that SIRT3 might have some effect on undifferentiated neurospheres.

Because SIRT3 is involved in a cell protective mechanism against oxidative stress, we examined the effect of this stress on the differentiation of neurospheres using antimycin A (AA), an inhibitor of mitochondrial respiratory chain complex III and a ROS inducer. Ten $\mathrm{nM}$ or $100 \mathrm{nM}$ AA was added to the differentiation medium and NPCs were cultured in it for 5 days. In the absence of AA, more than $90 \%$ of the neurospheres were spreading after 5 days in the differentiation medium indicating that differentiated cells migrated and extended protrusions. AA treatment dose-dependently inhibited spreading of neurospheres after differentiation (Fig. 3c). As shown in Fig. 3d, immunostaining of differentiated neurospheres gave rise to $\beta \mathrm{III}-$ tubulin ${ }^{+}, \mathrm{O}^{+}$and $\mathrm{GFAP}^{+}$cells, indicating that spheres differentiated into neurons, oligodendrocytes, and astrocytes, respectively (Fig. 3d; AA (-)). Treatment of NPCs with $10 \mathrm{nM}$ AA in the differentiation medium for 5 days faintly affected cell viability but reduced the number of $\beta$ III-tubulin ${ }^{+}$cells and $\mathrm{O}^{+}$cells after differentiation (Fig. 3d). Ten nM AA also suppressed extension of protrusions of $\beta$ III-tubulin ${ }^{+}$cells and $\mathrm{O}^{+}$cells (Fig. 3d). However, the number and shape of $\mathrm{GFAP}^{+}$cells were not affected by $10 \mathrm{nM}$ AA (Fig. 
3d). High concentrations of AA at $100 \mathrm{nM}$ severely reduced the number of survival cells in which double positive or triple positive cells against $\beta$ III-tubulin, GFAP, or O4 immunostaining appeared after differentiation (Fig. 3d). To examine a cell protective role of SIRT3, lentivirus-mediated knockdown of SIRT3 was introduced into NPCs and spheres were then differentiated in the medium for 5 days. As shown in Fig. 3e, SIRT3 knockdown reduced the number of survived cells and double positive or triple positive cells against $\beta$ III-tubulin, O4, or GFAP immunostaining appeared after differentiation (Fig. 3e).

\section{Ubiquitin-dependent degeneration of SIRT3ct protein.}

SIRT3ct protein expression levels were always weaker than those of SIRT3mt protein in the Western blot experiments (Fig. 1e,f). Low levels of SIRT3ct protein expression may be mediated by accelerated degradation of the protein in cells. Anisomycin is an inhibitor of protein synthesis. When protein synthesis was inhibited by anisomycin in COS7 cells $48 \mathrm{~h}$ after transfection of SIRT3ct-FLAG cDNA, the SIRT3ct-FLAG protein could not be detected after $1 \mathrm{~h}$ incubation with the drug. In contrast, SIRT3mt expression was not affected even after $2 \mathrm{~h}$ incubation with anisomycin (Fig. 4a). The SIRT3ct protein might be fragile and rapidly degraded by cellular proteases. We examined the effects of various protease inhibitors on SIRT3ct-FLAG protein levels in 
HEK293T cells. Treatment of cells with lactacystin, a proteasome inhibitor, increased the intensity of the SIRT3ct-FLAG band (Fig. 4b), whereas phenylmethylsulfonyl fluoride (PMSF) and leupeptin, inhibitors of serine, cysteine and/or threonine proteases, could not prevent degradation of SIRT3ct-FLAG (Fig. 4b). Pepstatin A, an inhibitor of acid proteases, failed to protect SIRT3ct-FLAG (Fig. 4b). A potent proteasome inhibitor MG132 significantly increased the expression level of SIRT3ct-FLAG but faintly affected the SIRT3mt-FLAG level (Fig. 4c). Since lactacystin and MG132 are proteasome inhibitors, SIRT3ct-FLAG may be degraded by a ubiquitin-dependent pathway. COS7 cells transfected with SIRT3ct-EGFP cDNA were immunostained with an anti-ubiquitin antibody. As shown in Fig. 4d, colocalization of SIRT3ct-EGFP with ubiquitin suggested that SIRT3ct was polyubiquitinated in cells. To detect ubiquitination of SIRT3ct, SIRT3ct-FLAG expressed in COS7 cells was immunoprecipitated with an anti-FLAG antibody and then analysed by an anti-ubiquitin antibody. Multiple bands larger than $28 \mathrm{kDa}$ of SIRT3ct-FLAG were detected, when cells were treated with MG132 (Fig. 4e). Thus, SIRT3ct-FLAG was ubiquitinated. The expression levels of SIRT3ct-FLAG and SIRT3ct-EGFP were faint in the absence of MG132 and were significantly enhanced by the treatment of cells with MG132 (Fig. 4g). SIRT3ct-FLAG and SIRT3ct-EGFP were proteins in which FLAG peptide and 
EGFP were fused in the $\mathrm{COOH}$-terminal end of SIRT3ct. Ubiquitin-dependent protein degradation was mediated by the ubiquitination of lysine residues or the $\mathrm{NH}_{2}$-terminal methionine residue of target proteins ${ }^{16}$. Because ubiquitination usually occurs in the amino group of lysine residues, we changed all of the 8 lysine residues of SIRT3ct to arginine residues in SIRT3ct-8KR-FLAG by site-directed mutagenesis (Supplementary Fig. 1). The expression level of SIRT3ct-8KR-FLAG was higher than that of SIRT3ctFLAG, but it was prominently increased by MG132 treatment, indicating that SIRT3ct8KR-FLAG was still degraded by a ubiquitin-proteasome system (Fig. 4f). Because ubiquitination of the $\mathrm{NH}_{2}$-terminal methionine residue of ERK3 is inhibited by a fusion of EGFP at the $\mathrm{NH}_{2}$-terminal end ${ }^{17}$, we examined the effect of EGFP fusion to SIRT3ctFLAG on the stability of SIRT3ct. EGFP-SIRT3ct-FLAG was the protein to which EGFP was fused at the $\mathrm{NH}_{2}$-terminal end of SIRT3ct-FLAG. We found that the expression of EGFP-SIRT3ct-FLAG was easily detected in the absence of MG132 (Fig. $4 \mathrm{~g}$ ), suggesting that the $\mathrm{NH}_{2}$-terminal methionine of SIRT3ct was a ubiquitination site. Treatment of cells expressing EGFP-SIRT3ct-FLAG with MG132 slightly enhanced the expression level of the protein compared with that in non-treated cells (Fig. 4g). When EGFP was introduced in the $\mathrm{NH}_{2}$-terminal region of SIRT3ct-8KR-FLAG, the intensity of the band of EGFP-SIRT3ct-8KR-FLAG was not enhanced by MG132 (Fig. 4h). In 
contrast, the expression level of EGFP-SIRT3ct-FLAG was slightly increased by MG132 treatment (Fig. 4h). These results suggested that the ubiquitination of the $\mathrm{NH}_{2}-$ terminal methionine residue mainly participated in the degradation of SIRT3ct and those in lysine residues of SIRT3ct partially contributed to SIRT3ct degradation.

\section{SIRT3ct as well as SIRT3mt protected cells against oxidative stress.}

SIRT3 plays a role in the suppression of reactive oxygen species (ROS) ${ }^{1,5}$. Stress inducer SP enhanced SIRT3ct expression in the cytoplasm and nucleus (Fig. 1f). We examined the effect of $\mathrm{H}_{2} \mathrm{O}_{2}$ on the expression and subcellular localization of SIRT3 in pheochromocytoma PC12 cells. Sirt3 mRNA expression levels were increased by $\mathrm{H}_{2} \mathrm{O}_{2}$ treatment (Fig. 5a). Immunostaining of endogenous SIRT3 in PC12 cells showed increased expression levels of SIRT3 by $\mathrm{H}_{2} \mathrm{O}_{2}$ treatment (Fig. 5b).

FOXO4, a transcription factor, has a cell protective role in response to oxidative stress and induces ROS-detoxifying enzymes such as SOD2 ${ }^{18}$. Treatment of cells with $\mathrm{H}_{2} \mathrm{O}_{2}$ enhanced the expression of SIRT3 and induced nuclear FOXO4 expression. Moreover, some cells showed nuclear costaining of SIRT3 and FOXO4 (Fig. 5b). Treatment of cells with AA also enhanced the expression of SIRT3 (Fig. 5b). Expression of SIRT3ct-EGFP in COS7 cells showed cytoplasmic localization of SIRT3ct-EGFP, where SIRT3ct-EGFP presented a dot-like appearance (Fig. 5c, bottom 
left panel). Compared to that of untreated cells, treatment of cells with $\mathrm{H}_{2} \mathrm{O}_{2}$

significantly enhanced the fluorescence intensity of SIRT3ct-EGFP in the cytoplasm of cells where SIRT3ct-EGFP was uniformly distributed (Fig. 5c, bottom right panel). We found that SIRT3ct-EGFP fluorescence was also detected in the nuclei of cells treated with $\mathrm{H}_{2} \mathrm{O}_{2}$ (Fig. 5c, bottom right panel). $\mathrm{H}_{2} \mathrm{O}_{2}$ treatment slightly enhanced the fluorescence level of SIRT3mt-EGFP in COS7 cells and was also induced nuclear localization of SIRT3mt-EGFP (Fig. 5c, upper panels). Nuclear localization and an increase of SIRT3 immunostaining by $\mathrm{H}_{2} \mathrm{O}_{2}$ were detected in the primary cultured neuronal cells isolated from the newborn mouse brain (Fig. 5d). More than $40 \%$ of cells showed nuclear SIRT3 immunostaining by $\mathrm{H}_{2} \mathrm{O}_{2}$ stimulation (Fig. 5d). Since SIRT3 has been reported to deacetylate histones ${ }^{8}$, it may also be possible that nuclear localization of SIRT3ct and SIRT3mt by $\mathrm{H}_{2} \mathrm{O}_{2}$ induces deacetylation of histone $\mathrm{H} 3$. Accordingly, in the presence of $\mathrm{H}_{2} \mathrm{O}_{2}$, overexpression of SIRT3ct-FLAG or SIRT3mt-FLAG significantly induced the deacetylation of histone H3 in COS7 cells (Fig. 5e).

Overexpression of SIRT3 increases SOD2 expression level by deacetylation and activation of $\mathrm{FOXO}^{19}$. Using PC12 cells, we found that SOD2 immunostaining was enhanced by SIRT3ct-EGFP expression (Fig. 5f). These observations suggested that 
SIRT3ct stability was increased by oxidative stress, and that SIRT3ct translocated into the nucleus and modulated gene expression.

SIRT3 has been shown to decrease ROS levels and inhibit cell death by $\operatorname{ROS}^{1,5}$. We examined the effect of the overexpression of SIRT3ct on $\mathrm{H}_{2} \mathrm{O}_{2}$-induced cell death and compared the result with that of SIRT3mt in COS7 cells. To identify transfected cells, we co-expressed the GFP protein with SIRT3ct or SIRT3mt. Using a pIRES-hrGFP vector, GFP was expressed separately from SIRT3 to eliminate an artificial effect that could result from the fusion of GFP to SIRT3. We found that the overexpression of SIRT3ct significantly reduced the number of dead cells under $\mathrm{H}_{2} \mathrm{O}_{2}$ treatment (Fig. 5g). Similar to the anti-apoptotic effect of SIRT3ct, the overexpression of SIRT3mt also decreased cell death induced by $\mathrm{H}_{2} \mathrm{O}_{2}$ (Fig. $5 \mathrm{~g}$ ). The potency of the cell-survival effect of SIRT3ct and that of SIRT3mt was similar under the present condition. Both SIRT3ct and SIRT3mt could participate in a cellular protective function under an oxidative stress condition.

\section{A $\beta$-dependent cell death was enhanced by SIRT3 knockdown and ameliorated by}

\section{SIRT3ct overexpression in PC12 cells.}

Extracellular accumulation of $\mathrm{A} \beta$ peptides is a pathological hallmark of Alzheimer's disease $^{20} . \mathrm{A} \beta$ peptides induce neuronal cell death via various pathways including 
increase of ROS levels ${ }^{20}$. A $\beta$ peptide 25-35 $\left(\mathrm{A} \beta_{25-35}\right)$ is shown to increase ROS level and cell death in PC12 cells ${ }^{21}$. To examine whether SIRT3 participates in a cell protective role against A $\beta$ peptides, we examined the effect of SIRT3 knockdown on cell death induced by $\mathrm{A} \beta_{25-35}$ treatment in PC12 cells. Both Sirt3-siRNA1 and Sirt3siRNA2 significantly reduced the expression levels of Sirt3 $m R N A$ and the SIRT3 protein in PC12 cells (Fig. 6 a,b). Knockdown of SIRT3 with Sirt3-siRNA2 alone increased the number of dead cells, which were significantly increased by $A \beta_{25-35}$ treatment (Fig. 6c). In the presence of $A \beta_{25-35}$, SIRT3 knockdown increased the number of dead cells by 2.5 -fold compared with those transfected with control-siRNA (Fig. 6c). To examine whether overexpression of SIRT3ct might rescue PC12 cells exposed to $\mathrm{A} \beta_{25-35}$, cells were transfected with SIRT3ct cDNA or the control plRES-hrGFP vector and were treated with $A \beta_{25-35 .} A \beta_{25-35}$ induced cell death and nearly $25 \%$ of cells treated with $A \beta_{25-35}$ died (Fig. 6d). Overexpression of SIRT3ct significantly reduced the number of dead cells and about $12 \%$ of cells died through $A \beta_{25-35}$ treatment. Thus, overexpression of SIRT3ct protected cells against the A $\beta$ toxicity and about $50 \%$ of cells escaped from death under the $A \beta_{25-35}$ treatment (Fig. 6d).

\section{Discussion}


Our experiments using cDNA cloning suggested that Sirt3ct $m R N A$ was highly expressed compared to Sirt3mt $m R N A$ in the brain, but SIRT3ct protein expression was much less than that of SIRT3mt. We found that SIRT3ct was the target of ubiquitindependent degradation and that the majority of SIRT3ct was degraded through ubiquitination of the $\mathrm{NH}_{2}$-terminal methionine residue. Proteins such as $\mathrm{MyoD}$, p21 and ERK3 are degraded by the $\mathrm{NH}_{2}$-terminal ubiquitination, which can escape through a fusion of large tags or EGFP at the $\mathrm{NH}_{2}$-terminal end ${ }^{16,17,22}$. Fusion of EGFP into SIRT3ct at the $\mathrm{NH}_{2}$-terminal region inhibited SIRT3ct degradation (Fig. 4f-h). Akimov et al. examined $\mathrm{NH}_{2}$-terminal ubiquitination of proteins and found that 104 proteins were ubiquitinated at the $\mathrm{NH}_{2}$-terminal methionine and they harbored either a proline or valine at the second position of their amino terminal sequences ${ }^{23}$. On a consistent basis, the amino acid residue at the second position of mouse and human SIRT3ct was valine. Acetylation of the $\mathrm{NH}_{2}$-terminal methionine likely competes with the $\mathrm{NH}_{2}$-terminal ubiquitination and proteins acetylated at the $\mathrm{NH}_{2}$-terminal methionine prefer alanine, glutamic acid and serine at the second amino acid position in their sequences ${ }^{23}$. SIRT3mt was resistant against proteasome degradation, since proteasome inhibitor MG132 had little effect on the stability of SIRT3mt (Fig. 4c). Because the second amino acid residue of mouse and human SIRT3mt was alanine (Fig. 1b), the amino 
terminal end of SIRT3mt might have been acetylated and able to escape from protein degradation. Although many proteins are ubiquitinated at the $\mathrm{NH}_{2}$-terminal end, the stability of these proteins is usually not affected by the ubiquitination ${ }^{23}$. Similar to p21, ERK3 and MyoD, SIRT3ct was an exception to the rule. p21 and ERK3 are degraded by the $\mathrm{NH}_{2}$-terminal ubiquitination but not by the ubiquitination of lysine residues, and only MyoD is degraded by both the ubiquitination at $\mathrm{NH}_{2}$-terminal methionine and lysine residues ${ }^{16,17,22}$. Since MyoD is a short-lived protein with a half-life of 30-60 $\min ^{24}$, ubiquitin-dependent degradation through $\mathrm{NH}_{2}$-terminal methionine and lysine residues of SIRT3ct may result in the short lifespan of SIRT3ct (Fig. 4a). At present, it is unknown whether the short half-life of SIRT3ct has any physiological significance.

SIRT3ct was promptly degraded in a control condition but we showed that a nongenotoxic stressor SP enhanced the expression of SIRT3ct in the cytoplasm and in the nucleus (Fig. 1f). The immunofluorescence level of SIRT3ct-EGFP was enhanced by $\mathrm{H}_{2} \mathrm{O}_{2}$ treatment (Fig. 5c) and immunostaining also showed an increased level of endogenous SIRT3 by oxidative stress in PC12 cells and in cultured neural cells (Fig. 5 b,d). Although Sirt3 $m R N A$ expression was enhanced by oxidative stress (Fig. 5a), enhancement of the SIRT3ct level expressed with SIRT3 cDNA by $\mathrm{H}_{2} \mathrm{O}_{2}$ treatment (Fig. 5c) suggested that ubiquitin-dependent degradation of SIRT3ct might be regulated 
by cellular stress. However, further study is needed to clarify the effect of stress signals on SIRT3ct stability. SIRT3ct reduced cell death induced by oxidative stress, in which the cell survival effect of SIRT3ct was comparable with that of SIRT3mt in COS7 cells (Fig. 5g). These experiments showed that SIRT3ct could participate in cell survival under stress condition, which was consistent with the role of SIRT3mt on cellular stress $^{1,5}$.

Most mitochondrial proteins are synthesized in the cytoplasm with $\mathrm{NH}_{2}$-terminal mitochondrial targeting sequences that enable the transport of the protein into mitochondria, in which MPP then cleaves the extension sequences in the matrix of mitochondria. A comparison of the $\mathrm{NH}_{2}$-terminal sequence of mouse SIRT3mt with that of human SIRT3mt suggested that the amino acid sequence of 1-37 of mouse SIRT3mt might be the signal for the transport into mitochondria (Fig. 1b). After cleavage of the mitochondrial targeting sequence by MPP, some of the mitochondrial proteins are further processed by the mitochondrial intermediate peptidase, which removes eight amino acids at the $\mathrm{NH}_{2}$-terminal region of the protein ${ }^{14}$. Overexpression of SIRT3mt gave three bands (37, 33 and $32 \mathrm{kDa}$ ) (Fig. 1e). If the full length SIRT3mt protein with amino acid sequences of 1-334 (37 kDa) was sequentially processed by MPP and mitochondrial intermediate peptidase, the intermediate size and the smallest size of 
SIRT3mt proteins could consist of amino acid sequences of 38-334 (33 kDa) and 46$334(32 \mathrm{kDa})$, respectively. Accordingly, appropriate sizes of SIRT3mt proteins were identified by our immunoblot experiments (Fig. 1e,f and 4c).

SIRT3ct and SIRT3mt dominantly localized in cytoplasm and mitochondria, respectively. However both SIRT3ct and SIRT3mt could localize in the nucleus under stress condition (Fig. 1 e,f). Overexpression of SIRT3mt promoted the deacetylation of histone $\mathrm{H} 3$ to the similar levels to that of SIRT3ct (Fig. 5e), indicating that SIRT3mt was also recruited to the nucleus. SIRT3 may be a nucleocytoplasmic shuttling protein similar to SIRT1, which has the nuclear localization signal (NLS) and nuclear export signal (NES) in its amino acid sequence ${ }^{25}$. Proteins with molecules smaller than 25-40 $\mathrm{kDa}$ passively diffuse across the nuclear membranes ${ }^{26}$. Because molecular weights of nuclear SIRT3mt and nuclear SIRT3ct were estimated to be $32 \mathrm{kDa}$ and $28 \mathrm{kDa}$, respectively, SIRT3 may be passively transported into the nucleus. Alternatively, efficient transport and export of a protein into the nucleus need NLS and NES in its amino acid sequences ${ }^{26}$. In the $\mathrm{COOH}$-terminal region of mouse SIRT3, we found a cluster of hydrophobic amino acids, i.e. PRLLINRDLVGP, and a stretch of negatively charged amino acids, i.e. PRRKDVV, which showed some similarity to those of NES and $\mathrm{NLS}^{27}$, respectively. Although similar sequences of these putative NES and NLS 
were found in human SIRT3, further studies are necessary to elucidate the regulation of subcellular localization of SIRT3.

We showed that differentiation of NPCs was severely affected by AA or SIRT3 knockdown (Fig. 3c-e). SIRT3 is necessary for the differentiation of osteoblasts. SIRT3 deficient mice show osteopenia with osteoblast dysfunction, whereas overexpression of SIRT3 or SOD2 improves the differentiation capability of primary osteoblasts derived from SIRT3-deficient mice ${ }^{28}$. Because SOD2 knockdown also suppresses osteoblast differentiation $^{28}$, oxidative stress may be involved in the disturbance of osteoblast differentiation by SIRT3 knockdown. Overexpression of SIRT3 in fact induces SOD2 via FOXO activation ${ }^{19}$ and SIRT3 has been shown to deacetylate and activate SOD2 ${ }^{3}$.

Weir et al. has reported that overexpression of SIRT3mt in primary cultured hippocampal neurons protects the neurons against ROS induced by $\mathrm{AA}^{13}$. We showed that overexpression of SIRT3ct reduced cell death induced by A $\beta$ in PC12 cells (Fig. 6d). Sirt3 $m R N A$ levels of brain increase in model mice and human patients of Alzheimer's disease ${ }^{13}$ and ROS levels significantly correlate with cognitive behaviour in model mice with Alzheimer's disease ${ }^{29,30}$. These results suggest that both SIRT3ct and SIRT3mt are involved in neuroprotection against A $\beta$ toxicity in Alzheimer's disease via ROS reduction. Since Sirt3 $m R N A$ and SIRT3 protein were constantly and 
highly expressed in the brains of various ages (Fig. 2), SIRT3ct as well as SIRT3mt might contribute to maintain brain homeostasis throughout the lifespan of both healthy and diseased brains.

\section{Materials and Methods}

Animals. Slc:ddY and C57BL/6J mice were purchased from Nihon SLC (Hamamatsu, Japan) and Sankyo Labo Service (Tokyo, Japan), respectively.

Cloning and expression of SIRT3 cDNAs. A lambda zap mouse brain cDNA library (Agilent Technologies, Santa Clara, CA, USA) was screened using a mouse SIRT3 cDNA fragment corresponding to nucleotides 312-635 in the coding region of mouse SIRT3 cDNA (NM_001177804.1). Total RNA was isolated with RNeasy (QIAGEN, Hilden, Germany) and cDNA was synthesized with SuperScript III reverse transcriptase (Thermo Fisher Scientific, Waltham, MA, USA). The cDNA fragment was obtained by RT-PCR of the total RNA from the mouse brain using a primer set of 5'gcaaccttcagcagtatgac-3' and 5'-ccatcacatcagcccatatg-3'. For the expression of SIRT3 cDNA, the coding regions of mouse SIRT3 cDNAs were subcloned in plRES-hrGFP (Agilent Technologies) or EGFP plasmids (Takara Bio, Kyoto, Japan). A QuikChange XL Site-Directed Mutagenesis Kit (Agilent Technologies) was used for site-directed mutagenesis as described previously ${ }^{31}$. 
Northern blot analysis. FirstChoice Northern Blot Mouse Blot 1 (Thermo Fisher Scientific), Mouse Embryo Stage Blot (Seegene, Songpa-Gu, Seoul, Republic of Korea) and Mouse Brain Aging Blot (Seegene) were probed with ${ }^{32} \mathrm{P}$-labeled cDNA fragments of SIRT3 and $\beta$-actin as described previously ${ }^{32}$.

Western blot analysis. Transfected cells were homogenized and analysed by Western blot analysis as described previously ${ }^{25}$.

Antibodies. Anti-acetylated histone H3 (382158), anti- $\alpha$-tubulin (T5168), anti- $\beta$ IIItubulin (AB9354), anti-histone H3 (H0164), anti-GAPDH (G8795) antibodies from Sigma-Aldrich (St. Louis, MO, USA), anti-lamin A/C (\#2032), anti-ubiquitin (\#3933) and anti-SOD2 (\#13141) antibodies from Cell Signaling Technology (Danvers, MA, USA) were used. Additionally, anti-cytochrome C (CTC03) from Thermo Fisher Scientific, anti-nestin (MAB353) from Merk (Danvers, MA, USA) were also used. Rabbit and mouse anti-FLAG (DYKDDDDK) antibodies were from Rockland (600401-383, Limerick, PA, USA) and FUJIFILM Wako Pure Chemical Corporation (Osaka, Japan), respectively. Anti-cytoplasmic aspartate aminotransferase (cAST) ${ }^{33}$, anti-mitochondrial aspartate aminotransferase $(\mathrm{mAST})^{33}$, and anti-SIRT1 ${ }^{32}$ antibodies were also used. The anti-SIRT3 antibody was raised in a rabbit against a synthetic 
peptide corresponding to the $\mathrm{COOH}$-terminal 16 amino acid residues

(LDLMQREPGKLDGQDR) of mouse SIRT3.

Cell Culture and Transfection. PC12 cells and COS7 cells were cultured in Dulbecco's modified Eagle's medium (FUJIFILM Wako Pure Chemical Corporation) supplemented with a $1 \%$ antibiotic-antimycotic mixed stock solution (Nacalai Tesque, Kyoto, Japan) and 10\% fetal bovine serum (MP Biomedicals, Irvine, CA, USA). ViaFect (Promega, Madison, WI, USA) or Lipofectamine 3000 (Thermo Fisher Scientific) was used for the transfection of plasmid DNAs and RNAiMAX Transfection Reagent (Thermo Fisher Scientific) was used for transfection of siRNAs. $50 \mathrm{nM}$ siRNAs were used for transfection. Sirt3-siRNAs were a duplex of 5'cucucaacagcuacauucatt-3' and 5'-ugaauguagcuguugagagtt-3' (Sirt3-siRNA1), and a duplex of 5'-gcuguaucuggcaugaauatt-3' and 5'-uauucaugccagauacagctt-3' (Sirt3siRNA2) (Cosmo Bio, Tokyo, Japan). For control siRNA experiments, mixtures of negative control-siRNA (Cosmo Bio) were used. Knockdown of SIRT3 was monitored by RT-PCR. Primer sets for RT-PCR were as follows: 5'-tagtccagggtgtggaaagg-3' and 5'-ccgcaggtgaagaagtaagc-3' for Sirt3, 5'-aatatatcccggacagttc-3' and 5'aagacaatctctggcttca-3' for Sirt1, and 5'-accacagtccatgccatcac-3' and 5'tccaccacctgttgctgta-3' for Gapdh. NPCs were isolated from the embryonic Scl:ddY 
mouse brain and cultured as neurospheres in a media hormone mix (MHM) medium containing EGF (20 ng/ml) and bFGF (20 ng/ml) as described previously ${ }^{15}$. For differentiation, neurospheres were plated onto poly-L-ornithine-coated microcoverslips and incubated for 5 days in a growth factor-free MHM medium containing 1\% FBS (differentiation medium). In some experiments, AA was added in the differentiation medium and spheres were incubated for 5 days. To estimate differentiation, the numbers of round spheres and flat-shaped differentiated neurospheres with migrating cells were counted in ten visual fields and compared. Mouse cortical neurons were cultured from the embryonic Scl:ddY mouse brain as described by Brewer et al. ${ }^{34}$. Cortical neurons were plated on poly-D-lysine-coated glass discs and cultured with a neurobasal medium containing 2\% B-27 Plus Supplement (Thermo Fisher Scientific) for 5 days. For the knockdown of SIRT3 in NPCs, the Sirt3-shRNA lentivirus or control-shRNA lentivirus were used to infect NPCs one day before differentiation ${ }^{33,35}$.

Treatment of cells with staurosporine, $\mathrm{H}_{2} \mathrm{O}_{2}$ and $\mathbf{A} \boldsymbol{\beta}$. COS7 cells was transfected with SIRT3mt-FLAG or SIRT3ct-FLAG cDNA and incubated for $24 \mathrm{~h}$ and were then exposed to $100 \mathrm{nM}$ staurosporine for $12 \mathrm{~h}$. The cells were homogenized and fractionated into nuclear and extra-nuclear fractions with Nuclear Extract Kit (40010, ACTIVE MOTIF, Carlsbad, CA, USA). PC12 cells were transfected with 
SIRT3ct:hrGFP or pIRES-hrGFP plasmid and incubated for $24 \mathrm{~h}$ and were exposed to $100 \mu \mathrm{M} \mathrm{H}_{2} \mathrm{O}_{2}$ or $50 \mu \mathrm{M} \mathrm{A} \beta_{25-35}$ in DMEM containing $10 \%$ serum for $24 \mathrm{~h}$. The cells were stained with Hoechst 33342 (DOJINDO, Kumamoto, Japan) to detect cell death. The numbers of cells expressing hrGFP with condensed or normal nuclei were counted from 5 visual fields of each treatment, and the percentage of dead cells was represented. Three independent experiments were carried out.

Immunostaining. Frozen sections of embryonic, postnatal or 8-week-old Scl:ddY mouse brains were immunostained as reported previously ${ }^{15}$. Alexa Fluor-labelled secondary antibodies (Thermo Fisher Scientific) were used and in some experiments the VECTASTAIN Universal Elite ABC PLUS kit (VECTASTAIN, Burlingame, CA, USA) was used. Mitochondria were stained by MitoTracker Red (M7512, Thermo Fisher Scientific) or an anti-cytochrome $\mathrm{C}$ antibody and nuclei were detected by Hoechst 33342.

Reagents. Anisomycin, antimycin A (AA) and phenylmethylsulfonyl fluoride (PMSF) were from Sigma-Aldrich. Other chemicals and reagents were from FUJIFILM Wako Pure Chemical Corporation. Mitochondria Isolation Kit for Mammalian Cells (89874, Thermo Fisher Scientific), ProteoExtract subcellular proteome extraction kit (539790, 
Millipore) and Nuclear Extraction Kit (40010, ACTIVE MOTIF Carlsbad, CA, USA)

were used.

Statistics. Data were expressed as means \pm SEMs. Comparisons between multiple groups were made using one-way analysis of variance followed by a post hoc Tukey test. The difference was considered significant if $\mathrm{p}$ was $<0.05$.

\section{Data Availability}

The datasets generated and/or analysed during the current study are available from the corresponding authors on reasonable request.

\section{References}

1. Haigis, M. C. \& Sinclair, D. A. Mammalian sirtuins: biological insights and disease relevance. Annu. Rev. Pathol. 5, 253-295 (2010).

2. Schwer, B., North, B. J., Frye, R. A., Ott, M. \& Verdin, E. The human silent information regulator (Sir)2 homologue hSIRT3 is a mitochondrial nicotinamide adenine dinucleotide-dependent deacetylase. J. Cell Biol. 158, 647-657 (2002).

3. Tao, R. et al. Sirt3-mediated deacetylation of evolutionarily conserved lysine 122 regulates MnSOD activity in response to stress. Mol. Cell 40, 893-904 (2010). 
4. Schlicker, C. et al. Substrates and regulation mechanisms for the human mitochondrial sirtuins Sirt3 and Sirt5. J. Mol. Biol. 382, 790-801 (2008).

5. Kane, A. E. \& Sinclair, D. A. Sirtuins and NAD + in the development and treatment of metabolic and cardiovascular diseases Circ. Res. 123, 868-885 (2018).

6. Zhang, J. et al. Extended wakefulness: compromised metabolics in and degeneration of locus ceruleus neurons. J. Neurosci. 34, 4418-4431 (2014).

7. Cheng, A. et al. Mitochondrial SIRT3 mediates adaptive responses of neurons to exercise and metabolic and excitatory challenges. Cell Metab. 23, 128-142 (2016).

8. Scher, M. B., Vaquero, A., \& Reinberg, D. SirT3 is a nuclear NAD+-dependent histone deacetylase that translocates to the mitochondria upon cellular stress. Genes Dev. 21, 920-928, (2007).

9. Nakamura, Y., Ogura, M., Tanaka, D. \& Inagaki, N. Localization of mouse mitochondrial SIRT proteins: shift of SIRT3 to nucleus by co-expression with SIRT5. Biochem. Biophys. Res. Commun. 366, 174-179 (2008).

10. Cooper, H. M., Huang, J. Y., Verdin, E. \& Spelbrink, J. N. A new splice variant of the mouse SIRT3 gene encodes the mitochondrial precursor protein. PloS One 4, e4986 (2009). doi: 10.1371/journal.pone.0004986. 
11. Bao, J., Lu, Z. \& Joseph, J. J. Characterization of the murine SIRT3 mitochondrial localization sequence and comparison of mitochondrial enrichment and deacetylase activity of long and short SIRT3 isoforms. J. Cell Biochem. 110, 238247 (2010).

12. Iwahara, T., Bonasio, R., Narendra, V. \& Reinberg, D. SIRT3 functions in the nucleus in the control of stress-related gene expression. Mol. Cell. Biol. 32, 5022$5034(2012)$.

13. Weir, H. J. M. et al. CNS SIRT3 expression is altered by reactive oxygen species and in Alzheimer's disease. PloS One 7, e48225 (2012). doi: 10.1371/journal.pone.0048225.

14. Shimokata, K. et al. Substrate recognition by mitochondrial processing peptidase toward the malate dehydrogenase precursor. J. Biochem. 122, 1019-1023 (1997).

15. Hisahara, S. et al. Histone deacetylase SIRT1 modulates neuronal differentiation by its nuclear translocation. Proc. Natl. Acad. Sci. USA 105, 15999-15604 (2008).

16. Breitschopf, K., Bengal, E., Ziv, T., Admon, A. \& Ciechanover, A. A novel site for ubiquitination: the N-terminal residue, and not internal lysines of $\mathrm{MyoD}$, is 
essential for conjugation and degradation of the protein. EMBO J. 17, 5964-5973 (1998).

17. Coulombe, P., Rodier, G., Bonneil, E., Thibault, P. \& Meloche, S. N-Terminal ubiquitination of extracellular signal-regulated kinase 3 and p21 directs their degradation by the proteasome Actions. Mol. Cell Biol. 24, 6140-6150 (2004).

18. Hori, Y. S., Kuno A., Hosoda, R. \& Horio Y. Regulation of FOXOs and p53 by SIRT1 modulators under oxidative stress. PLoS One 8, e73875 (2013). doi.org/10.1371/journal.pone.0073875.

19. Sundaresan, N. R. et al. Sirt3 blocks the cardiac hypertrophic response by augmenting Foxo3a-dependent antioxidant defense mechanisms in mice. J. Clin. Invest. 119, 2758-2771 (2009).

20. Canter, R. G., Penney, J. \& Tsai, L. H. The road to restoring neural circuits for the treatment of Alzheimer's disease. Nature 539, 187-196 (2016).

21. Lin, Y. H. et al. Salvianolic acid B, an antioxidant from Salvia miltiorrhiza, prevents Abeta(25-35)-induced reduction in BPRP in PC12 cells. Biochem. Biophys. Res. Commun. 348, 593-599 (2006). 
22. Bloom, J., Amador, V., Bartolini, F., DeMartino, G. \& Pagano, M. Proteasomemediated degradation of p21 via N-terminal ubiquitinylation. Cell 115, 71-82 (2003).

23. Akimov, V. et al. UbiSite approach for comprehensive mapping of lysine and Nterminal ubiquitination sites. Nat. Struct. Mol. Biol. 25, 631-640 (2018).

24. Thayer, M. J. et al. Positive autoregulation of the myogenic determination gene MyoD1. Cell 58, 241-248 (1989).

25. Tanno, M., Sakamoto, J., Miura, T., Shimamoto, K. \& Horio, Y. Nucleocytoplasmic shuttling of $\mathrm{NAD}^{+}$-dependent histone deacetylase SIRT1. J. Biol. Chem. 282, 6823-6832 (2007).

26. Tran, E. J. \& Wente, S. R. Dynamic nuclear pore complexes: life on the edge. Cell 125, 1041-1053 (2006).

27. Xu, L. \& Massagué, J. Nucleocytoplasmic shuttling of signal transducers. Nat. Rev. Mol. Cell Biol. 5, 209-219 (2004).

28. Gao, J. et al. SIRT3/SOD2 maintains osteoblast differentiation and bone formation by regulating mitochondrial stress. Cell Death Differ. 25, 229-240 (2018). 
29. Matsumura, A. et al. Evaluation of oxidative stress in the brain of as transgenic mouse model of Alzheimer's disease by in vivo electron paramagnetic resonance imaging. Free Radic Biol Med 85, 165-173 (2015).

30. Saito, T. et al. Early administration of galantamine from preplaque phase suppresses oxidative stress and improves cognitive behavior in APPswe/PS1dE9 mouse model of Alzheimer's disease. Free Radic Biol Med 145, 20-32 (2019).

31. Kuno, A. et al. Resveratrol improves cardiomyopathy in dystrophin-deficient mice through SIRT1 protein-mediated modulation of p300 Protein. J. Biol. Chem. 288, 5963-5972 (2013).

32. Sakamoto, J., Miura, T., Shimamoto, K. \& Horio, Y. Predominant expression of Sir2 $\alpha$, an NAD-dependent histone deacetylase in the embryonic mouse heart and brain. FEBS Lett. 556, 281-286 (2004).

33. Kunimoto, R. et al. SIRT1 regulates lamellipodium extension and migration of melanoma cells. J. Invest. Dermatol. 134, 1693-1700 (2014).

34. Brewer, G. J., Torricelli, J. R., Evege, E. K. \& Price, P. J. Optimized survival of hippocampal neurons in B27-supplemented Neurobasal, a new serum-free medium combination. J. Neurosci. Res. 35, 567-576 (1993). 
35. Araki, T., Sasaki, Y. \& Milbrandt, J. Increased nuclear NAD biosynthesis and SIRT1 activation prevent axonal degeneration. Science 305, 1010-1013 (2004).

\section{Acknowledgements}

This work was supported by grants from the Japanese Society for the Promotion of

Science Grants-in-Aid for Scientific Research (\#15K08312, \#18K06965, 22590245, 15659062, 13035038), the Osaka Medical Research Foundation for Intractable Diseases and the Hiroshige Kondo Foundation.

\section{Author contributions}

T. H., T. M., S. H., A. K. and Y. H. designed the study. T. H., T. M., S. H., A. K., R. K., R. H., N. I., M. T. and Y. H executed the study. T. M., A. K., S. S. and Y. H. analysed the data. T. M., N. I. and Y. H. wrote the manuscript. All authors read and approved the final version of the manuscript.

Corresponding author

Correspondence to Yoshiyuki Horio

\section{Ethics declaration.}


The Animal Welfare Guidelines of Sapporo Medical University were followed in all of the studies and experiments using mice. Approval for the experiments was obtained from the Ethical Committee for the Care and Use of Laboratory Animals of Sapporo Medical University (approval numbers: 11-017 and 11-046).

Competing interests. The authors declare no competing interests.

\section{Figure legends}

Figure 1. Cytoplasmic and mitochondrial mouse SIRT3 proteins.

(a) A schematic diagram of mouse Sirt3 gene, Sirt3 mRNAs and SIRT3 proteins. Sirt3mt and SIRT3mt indicate mitochondrial Sirt3 $m R N A$ and the SIRT3 protein, respectively. Sirt3ct1 and Sirt3ct2 indicate Sirt3 mRNAs for the cytoplasmic SIRT3 (SIRT3ct) protein. Exons were indicated by numbers, in which exon 3A is used in Sirt3ctl and Sirt3ct2, and exon 3B is used in Sirt3mt mRNA. Open and closed triangles indicate start codon and stop codon, respectively. (b) Comparison of the $\mathrm{NH}_{2}$-teriminal amino acid sequence of mouse SIRT3mt with the $\mathrm{NH}_{2}$-terminal region of human SIRT3mt. Arginine $(\mathrm{R})$ and proline $(\mathrm{P})$ residues conserved in mitochondrial targeting sequences are indicated by red and blue text, respectively (left panel). A helical wheel plot of residues 18-35 of mouse SIRT3mt shows a cluster of positively charged arginine 
residues on one side of the helix opposed by hydrophobic amino acids on the other side, a typical feature of mitochondrial targeting sequences (right panel). Arginine residues and hydrophobic amino acid residues are indicated by red and blue text, respectively. (c) Comparison of mouse and human SIRT3ct and SIRT3mt. Identities between amino acid sequences of conserved regions are indicated with percentages (\%). Mitochondrial targeting sequence of residues 1-25 of human SIRT3mt and the corresponding region of mouse SIRT3mt are shown in red. (d) Distribution of SIRT3mt-EGFP and SIRT3ctEGFP in COS7 cells. Mitochondria are labelled with MitoTracker Red. Scale bar: 10 $\mu \mathrm{m}$. (e) COS7 cells expressing SIRT3mt-FLAG or SIRT3ct-FLAG were homogenized and fractionated into nuclear $(\mathrm{Nu})$, cytoplasmic $(\mathrm{Ct})$ and mitochondrial $(\mathrm{Mt})$ fractions and analysed by Western blot using an anti-FLAG antibody. The membrane filter was reblotted by antibodies of Lamin A/C, cAST and mAST, markers of nuclear, cytoplasmic and mitochondrial fractions, respectively. (f) COS7 cells expressing SIRT3mt-FLAG or SIRT3ct-FLAG were treated with a non-genotoxic stressor staurosporine $(100 \mathrm{nM})$ for $12 \mathrm{~h}$ and fractionated into nuclear $(\mathrm{Nu})$ and extra-nuclear (En) fractions. En fractions contain cytoplasm and mitochondria.

Figure 2. Expression of SIRT3 in the brain and neural precursor cells. 
(a-c) Northern blot analyses of Sirt3 mRNA: (a) Various organs. (b) Brains of various ages. (c) Fetuses of various stages. $28 \mathrm{~s}$ and $18 \mathrm{~s}$ indicate $28 \mathrm{~s}$ and $18 \mathrm{~s}$ units of ribosomal RNA, respectively. E: embryonic day, d: day, w: week, and m: month. (d) RT-PCR analysis of Sirt3 and Sirt1 in fetal and postnatal brains. (e) SIRT3 immunostaining (red) of embryonic and postnatal brains. P: postnatal day and V: ventricles. Scale bars: $50 \mu \mathrm{m}$ (E8.5), $20 \mu \mathrm{m}$ (E10.5 and E14.5) and $100 \mu \mathrm{m}$ (P2). (f) SIRT3 and SIRT1 immunostaining (green) of cultured neurospheres isolated from mouse embryonic brain. Nestin (red) is a marker of NPCs. Nuclei are stained with Hoechst 33342 (blue). Scale bar: $100 \mu \mathrm{m}$. (g) CA1 region of hippocampus, cerebral cortex, lateral ventricle and cerebellum were immunostained with an anti-SIRT3 antibody (brown). Scale bar: 50 $\mu \mathrm{m}$. (h) SIRT3 immunostaining (green) of cultured neural cells. MitoTracker Red (purple) and $\beta$ III-tubulin (blue) are markers for mitochondria and neural cells, respectively. Scale bar: $10 \mu \mathrm{m}$.

Figure 3. SIRT3 knockdown disturbed neural precursor cell differentiation.

(a) NPCs mechanically dissociated from cultured neurospheres were spread on poly-Lornithine-coated glasses and immunostained with an anti-SIRT3 (green) and an anticytochrome C (a marker of mitochondria, red) antibodies. Scale bar: $10 \mu \mathrm{m}$. (b) Expression of SIRT3 (green), SIRT1 (green) and $\beta$ III-tubulin (red) of undifferentiated 
neurospheres under proliferation conditions and differentiated neurospheres. Scale bar:

$50 \mu \mathrm{m}$. (c) Neurospheres were differentiated in the presence or absence of AA for 5 days. The number of neurospheres with dissociated cells as well as round undifferentiated neurospheres were counted and compared. ${ }^{*} \mathrm{p}<0.01$. (d) Neurospheres were differentiated with a differentiation medium in the presence or absence of AA for 5 days and immunostained with $\beta$ III-tubulin (red), O4 (an oligodendrocyte marker, green) and GFAP (an astrocyte marker, blue) antibodies. Scale bar: $50 \mu \mathrm{m}$. (e) Neurospheres were infected with lentiviruses expressing control-shRNA or Sirt3shRNA. One day after the infection, cells were differentiated in the differentiation medium for 5 days and then immunostained. In some experiments AA was added into the differentiation medium. Scale bars: $50 \mu \mathrm{m}$.

Figure 4. Ubiquitin-dependent degradation of SIRT3ct protein.

(a) COS7 cells transfected with SIRT3mt-FLAG or SIRT3ct-FLAG cDNA were treated with $10 \mu \mathrm{M}$ anisomycin for the indicated times and analysed by Western blot. The filter was probed with an anti-FLAG antibody and then rebotted with an anti-GAPDH antibody. (b) COS7 cells transfected with SIRT3ct-FLAG cDNA were treated with various protease inhibitors for $10 \mathrm{~h}$ and analysed with an anti-FLAG antibody. Lac: 2 $\mu \mathrm{M}$ or $10 \mu \mathrm{M}$ lactacystin, PM: $1 \mathrm{mM}$ PMSF, Leu: $100 \mu \mathrm{M}$ leupeptin and Pep: $100 \mu \mathrm{M}$ 
pepstatin. (c) HEK293T cells transfected with SIRT3mt-FLAG or SIRT3ct-FLAG cDNA were treated with $100 \mu \mathrm{M}$ MG132 for $10 \mathrm{~h}$ and analysed with an anti-SIRT3 antibody. (d) Immunostaining of ubiquitin (Ub, red) in COS7 cells transfected with SIRT3ct-EGFP cDNA. Scale bar: $20 \mu \mathrm{m}$. (e) COS7 cells transfected with SIRT3ctFLAG cDNA were incubated in the presence or absence of $100 \mu \mathrm{M}$ MG132 for $10 \mathrm{~h}$, homogenized, immunoprecipitated with anti-FLAG antibody-resin, and then analysed with an anti-ubiquitin antibody. The filter was reblotted with an anti-FLAG antibody. (f) COS7 cells transfected with SIRT3ct-FLAG or SIRT3ct-8KR-FLAG cDNA were incubated for $48 \mathrm{~h}$ and then treated with MG132 for $10 \mathrm{~h}$. Cells were homogenized and analysed by Western blot. (g) COS7 cells transfected with EGFP-SIRT3ct-FLAG, SIRT3ct-FLAG, or SIRT3ct-EGFP cDNA were incubated for $48 \mathrm{~h}$ and then treated with or without MG132 for $10 \mathrm{~h}$. Cells were analysed by an anti-GFP antibody and then sequentially reblotted with an anti-FLAG and an anti-GAPDH antibodies. Green and blue arrowheads indicate EGFP-fused SIRT3ct and FLAG-tagged SIRT3ct proteins, respectively. $*$ and $* *$ indicate non-specific bands by the an anti-GFP antibody and an anti-FLAG antibody, respectively. (h) COS7 cells transfected with SIRT3ct-FLAG, SIRT3ct-8KR-FLAG, EGFP-SIRT3ct-FLAG, or EGFP-SIRT3ct-8KR-FLAG cDNA 
were incubated for $48 \mathrm{~h}$ and treated with or without MG132 for $10 \mathrm{~h}$. Cells were homogenized and analysed by Western blot. nt: no transfection.

Figure 5. Nuclear translocation of SIRT3ct protected cells against oxidative stress. (a) PC12 cells were treated with $200 \mu \mathrm{M} \mathrm{H}_{2} \mathrm{O}_{2}$ for the indicated hours and analysed by RTPCR. (b) PC12 cells were treated with $100 \mu \mathrm{M} \mathrm{H}_{2} \mathrm{O}_{2}$ or $100 \mathrm{nM}$ antimycin A (AA) for $12 \mathrm{~h}$ and then analysed by immunostaining of SIRT3 (green) and FOXO4 (red). Arrows indicate nuclear staining of SIRT3 and FOXO4. Bar: $20 \mu \mathrm{m}$. (c) COS7 cells transfected with SIRT3mt-EGFP or SIRT3ct-EGFP cDNA were treated with or without $100 \mu \mathrm{M}$ $\mathrm{H}_{2} \mathrm{O}_{2}$ for $12 \mathrm{~h}$. Bar: $20 \mu \mathrm{m}$. (d) Mouse primary cortical neurons were treated with 500 $\mathrm{nM} \mathrm{H} \mathrm{H}_{2} \mathrm{O}_{2}$ for $12 \mathrm{~h}$ and stained with an anti-SIRT3 antibody (green), an anti- $\beta$ III-tubulin antibody (red) and Hoechst 33342 (blue). Bar: $50 \mu \mathrm{m}$. The numbers of cells expressing cytoplasmic SIRT3 and nuclear SIRT3 were counted and compared (right panel). $* \mathrm{p}<0.001$. (e) COS7 cells transfected with SIRT3mt-FLAG, SIRT3ct-FLAG cDNA or both plasmids were treated with $100 \mu \mathrm{M} \mathrm{H}_{2} \mathrm{O}_{2}$ and $25 \mathrm{nM}$ trichostatin $\mathrm{A}$, an inhibitor of $\mathrm{NAD}^{+}$-independent histone deacetylases, for $12 \mathrm{~h}$. The expression levels of acetylated histone $\mathrm{H} 3$ and total histone $\mathrm{H} 3$ were analysed by Western blot. The filter blotted with an anti-acetyl histone $\mathrm{H} 3$ and an anti-GAPDH antibodies was sequentially reblotted with an anti-histone $\mathrm{H} 3$ antibody and an anti-FLAG antibody. Intensities of bands were 
compared $(\mathrm{n}=3) . * \mathrm{p}<0.05, * * \mathrm{p}<0.01$. (f) PC12 cells transfected with SIRT3ct-EGFP cDNA were incubated for $48 \mathrm{~h}$ and then treated with $100 \mu \mathrm{M} \mathrm{H}_{2} \mathrm{O}_{2}$ for $12 \mathrm{~h}$. The cells were immunostained with an anti-SOD2 antibody (red). Nuclei were stained with Hoechst 33342. The arrow and arrowhead indicate SIRT3ct-EGFP ${ }^{+}$cells and SIRT3ct-

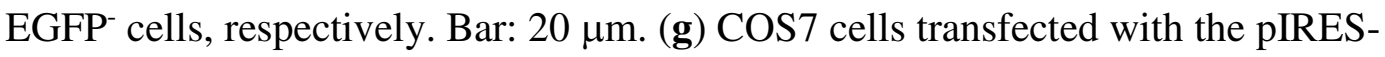
hrGFP vector, SIRT3mt:hrGFP or SIRT3ct:hrGFP plasmid, were incubated for $24 \mathrm{~h}$ and then treated with $100 \mu \mathrm{M} \mathrm{H}_{2} \mathrm{O}_{2}$ for $24 \mathrm{~h}$. Cells were then stained with Hoechst 33342 (blue). The numbers of hrGFP ${ }^{+}$cells with condensed nuclei and those of hrGFP ${ }^{+}$cells without condensed nuclei in a visual field were counted. Five visual fields were counted in each condition. The percentages of dead $\mathrm{hrGFP}^{+}$cells in total $\mathrm{hrGFP}^{+}$cells are shown (right panel). ${ }^{*} \mathrm{p}<0.05$. Scale bar: $20 \mu \mathrm{m}$.

Figure 6. SIRT3ct protected cells against $A \beta_{25-35}$ toxicity. (a,b) PC12 cells were transfected with control-siRNA, Sirt3-siRNA1 or Sirt3-siRNA2. Expression levels of Sirt3 mRNA (a) and SIRT3 protein (b) were compared after $48 \mathrm{~h}$ of transfection. **p<0.01. (c) PC 12 cells were transfected with control-siRNA or Sirt3-siRNA2 and then treated with $50 \mu \mathrm{M} \mathrm{A} \beta_{25-35} 24 \mathrm{~h}$ after transfection. Cells were further incubated for an additional $24 \mathrm{~h}$ and then stained with Hoechst 33342 to detect dead cells. Condensed apoptotic nuclei are indicated by arrowheads. (d) PC12 cells transfected with pIRES- 
hrGFP or SIRT3ct:hrGFP plasmid were incubated for $24 \mathrm{~h}$ and then treated with $50 \mu \mathrm{M}$ $A \beta_{25-35}$ for 24 h. Cells were stained with Hoechst 33342 to detect apoptotic cells.

Number of apoptotic hrGFP ${ }^{+}$cells were compared with those of $\mathrm{hrGFP}^{+}$cells. ${ }^{*} \mathrm{p}<0.05$, $* * \mathrm{p}<0.01$ 
Figure 1.

a

mouse Sirt3 gene

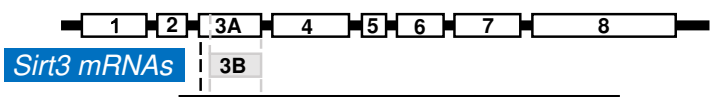

Sirt3mt

Sirt3ct1

Sirt3ct2

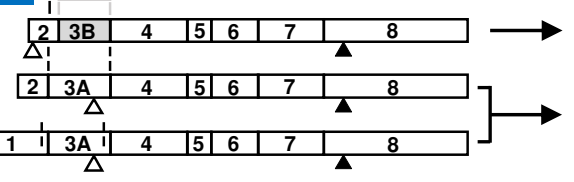

b

$\begin{array}{lllllll}\text { mouse } & 1 & 15 & 20 & 27 & 35 & 38\end{array}$

SIRT3mt MALDPLGAVVLQSIMAL SG - RLALAALRLWGPGGGRRP I S

human SIRT3mt

MA'FWGW' R-A'A'AALRL' '́' '́RV VERV EAG

C

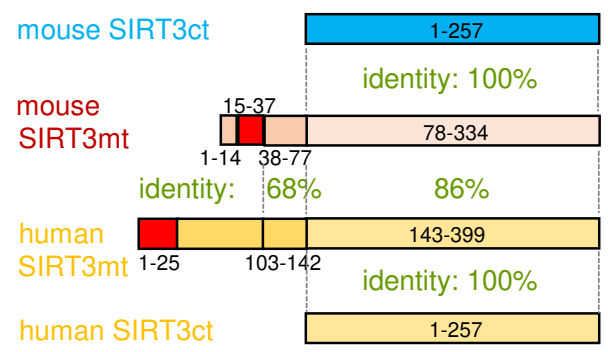

e

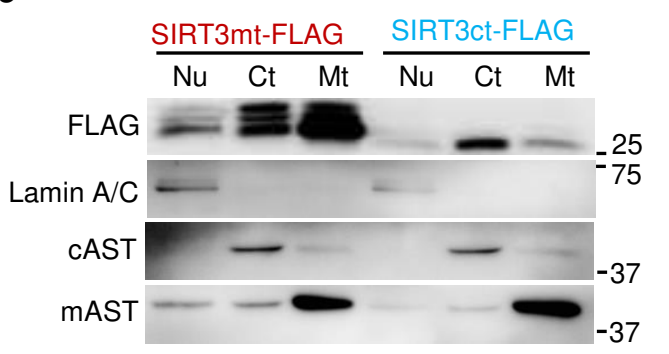

d
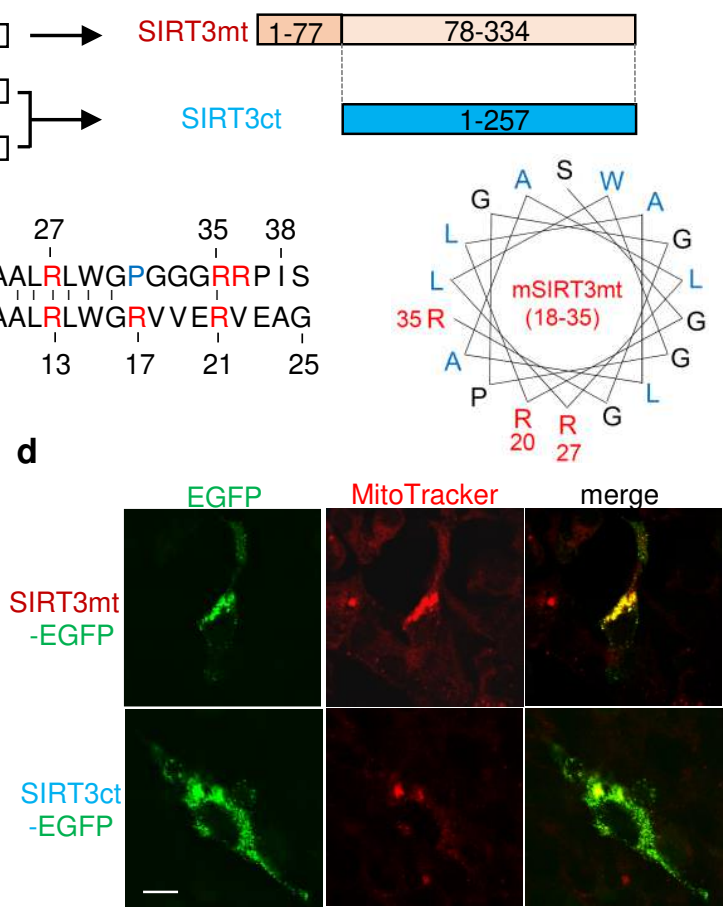

f

$\mathrm{SP} \frac{-}{\mathrm{Nu} \mathrm{En}} \frac{+}{\mathrm{Nu} \mathrm{En}} \frac{-}{\mathrm{Nu} \mathrm{En}} \frac{+}{\mathrm{Nu} \mathrm{En}}$

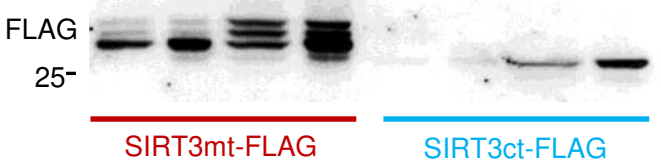


Figure 2.

a

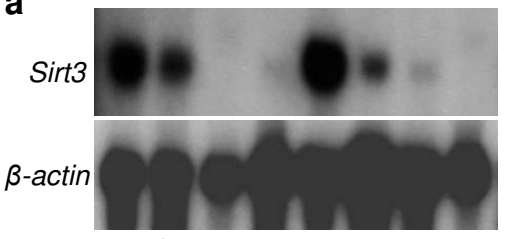

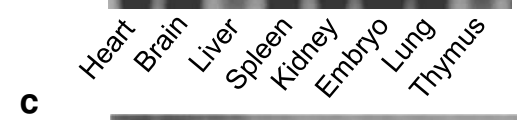

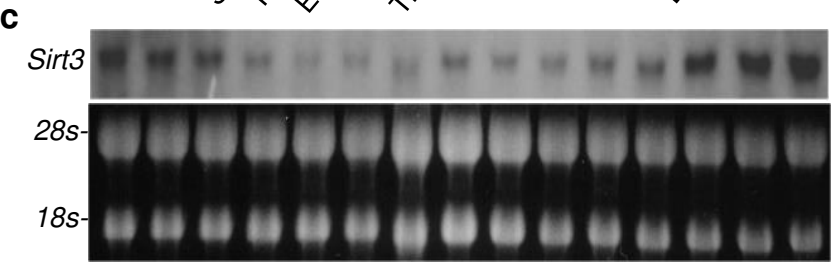

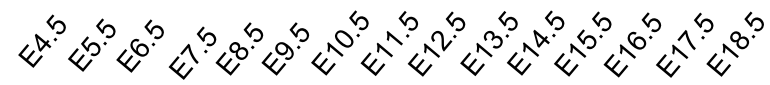

b

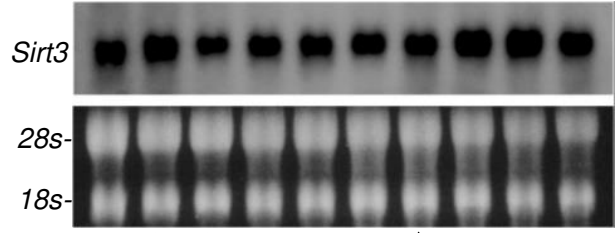

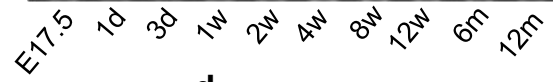

d

e

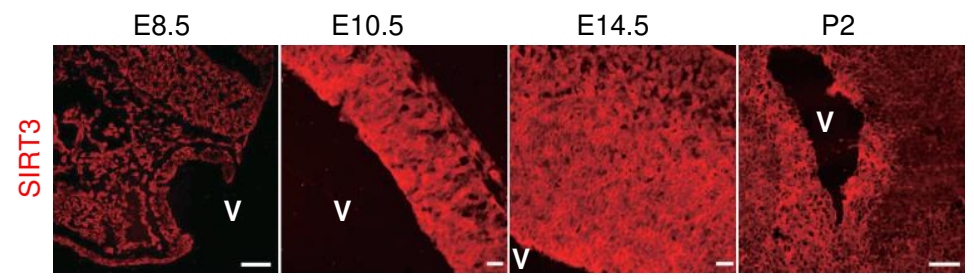

$\mathbf{f}$

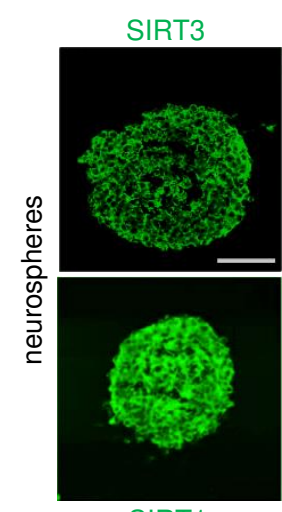

SIRT1

nestin

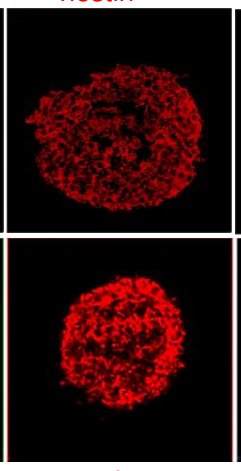

nestin

g

CA1

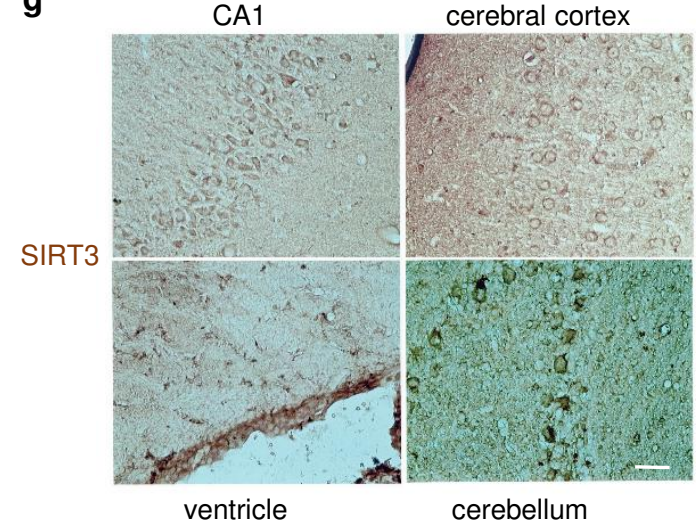

ventricle

nuclei

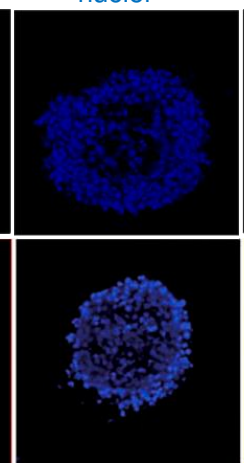

nuclei

h

BIII-tubulin

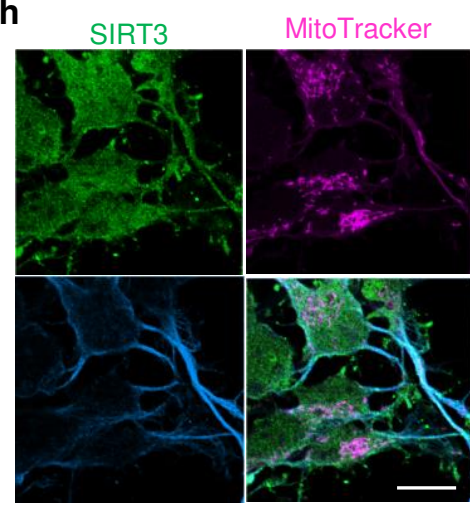

merge

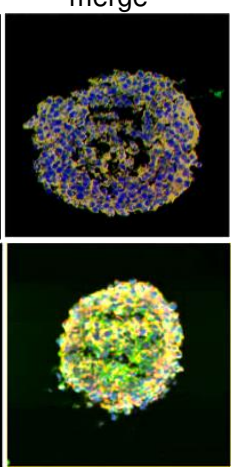

merge

merge

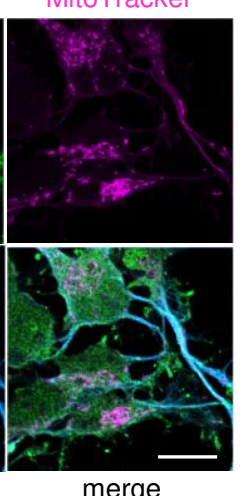


Figure 3.

a

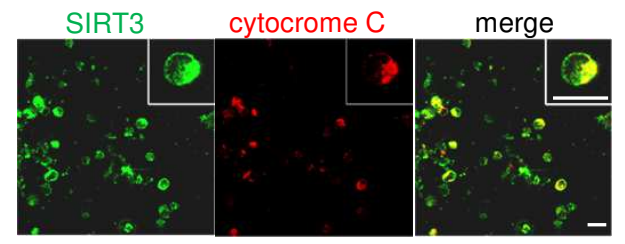

b

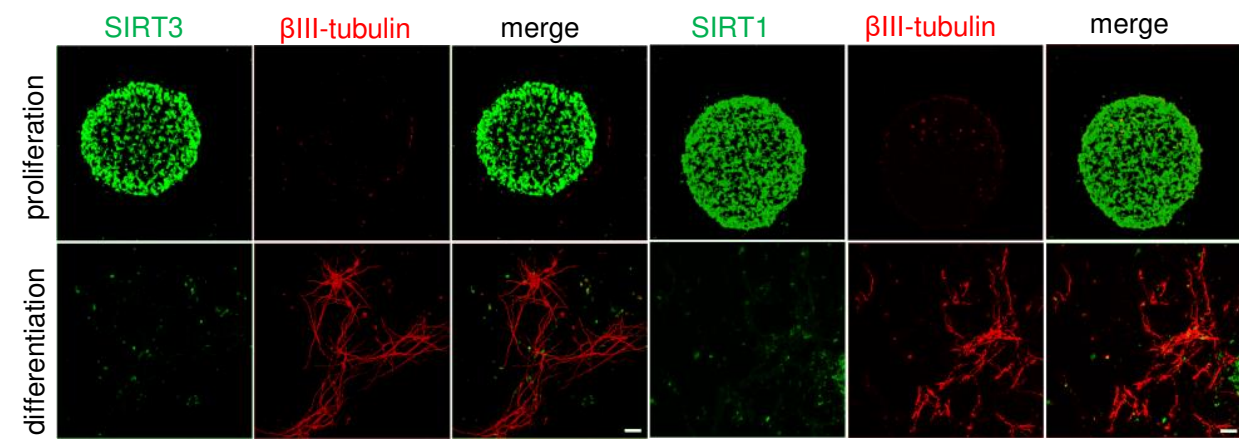

C

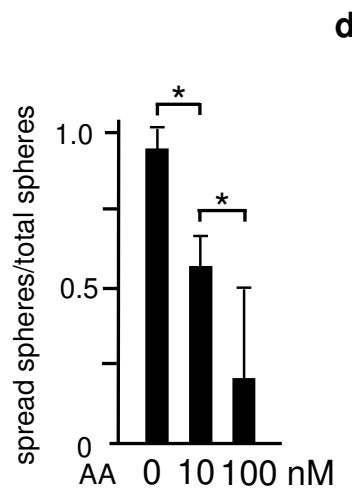

d

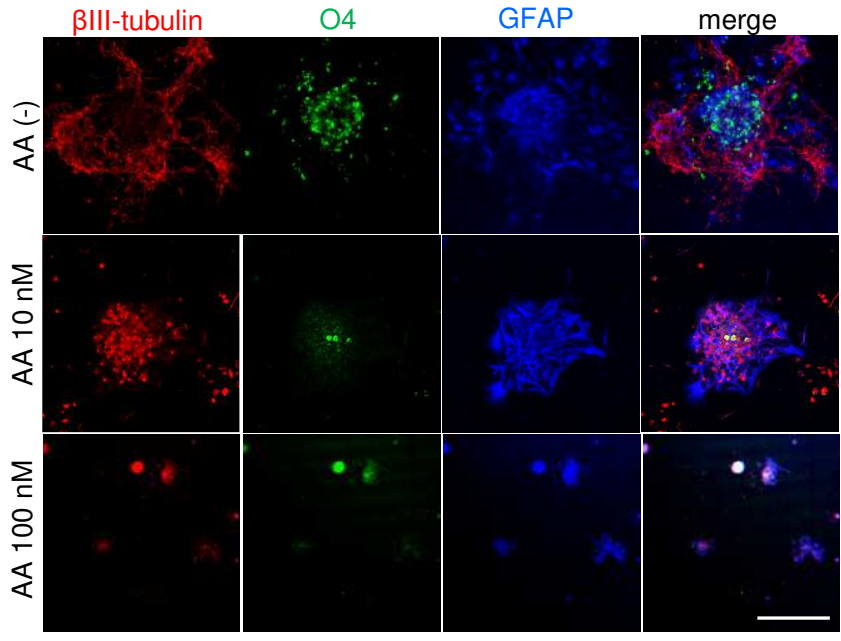

e

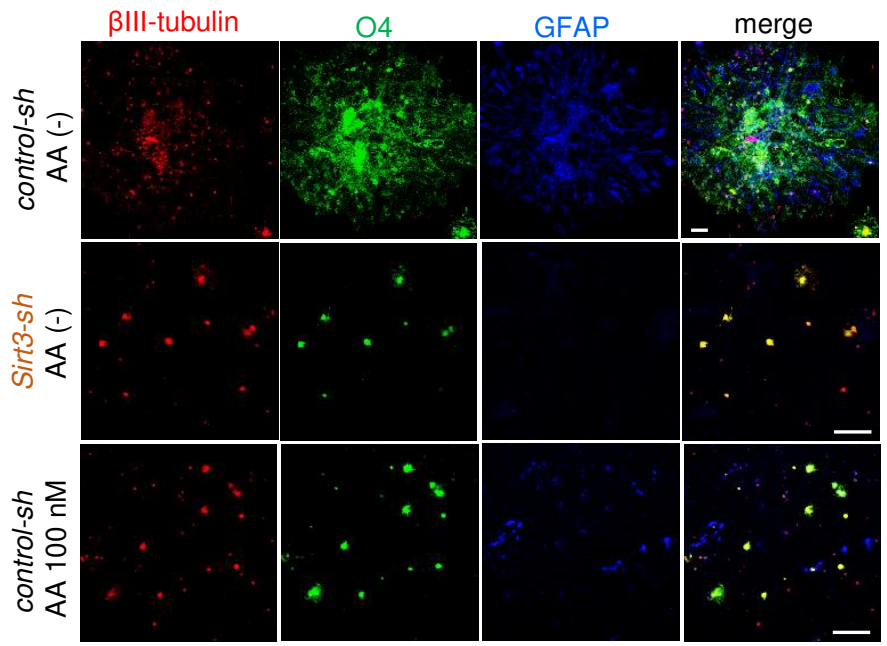


Figure 4.

a

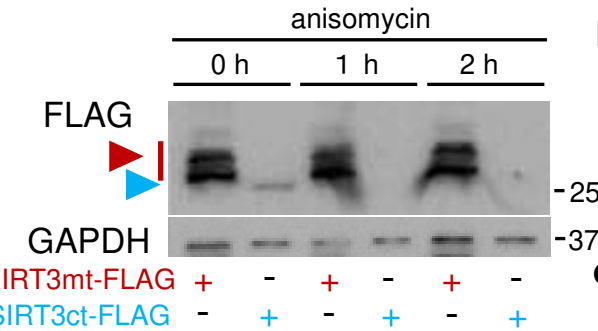

b

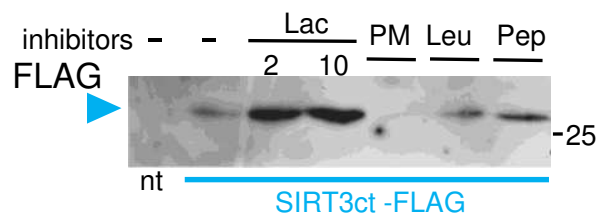

d SIRT3ct

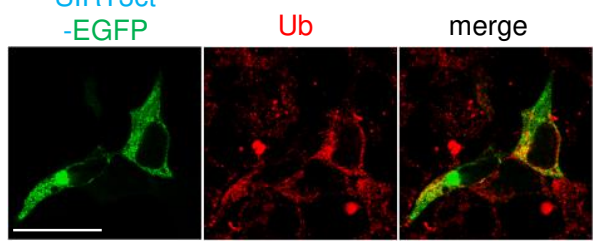

g

MG132 - $\quad-\quad-\quad+\quad+\quad+\quad+$

e
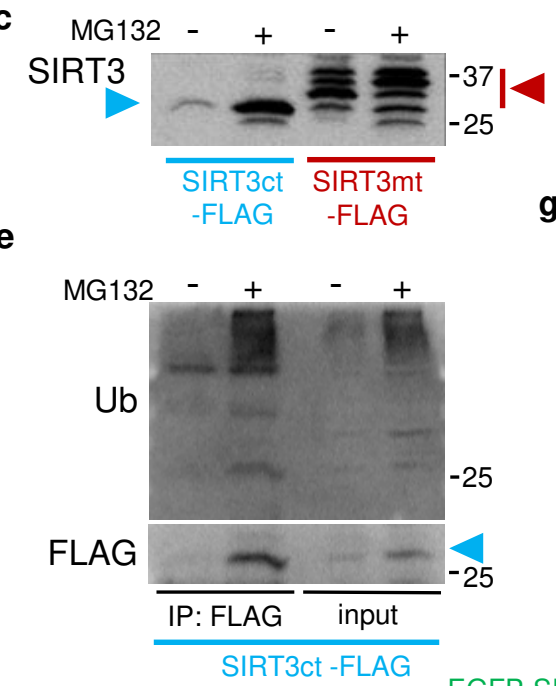

$-25$

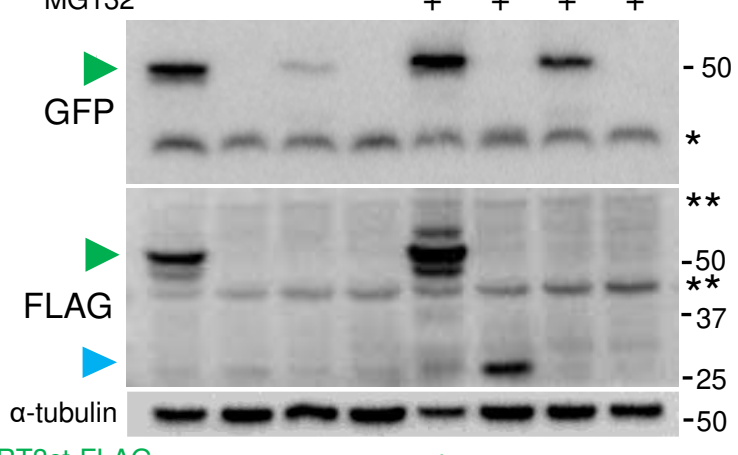

f

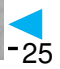

EGFP-

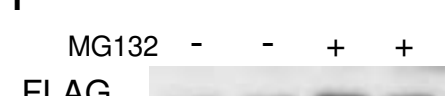

SIRT3ct -FLAG -

SIRT3ct -EGFP -

FLAG

h

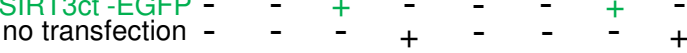
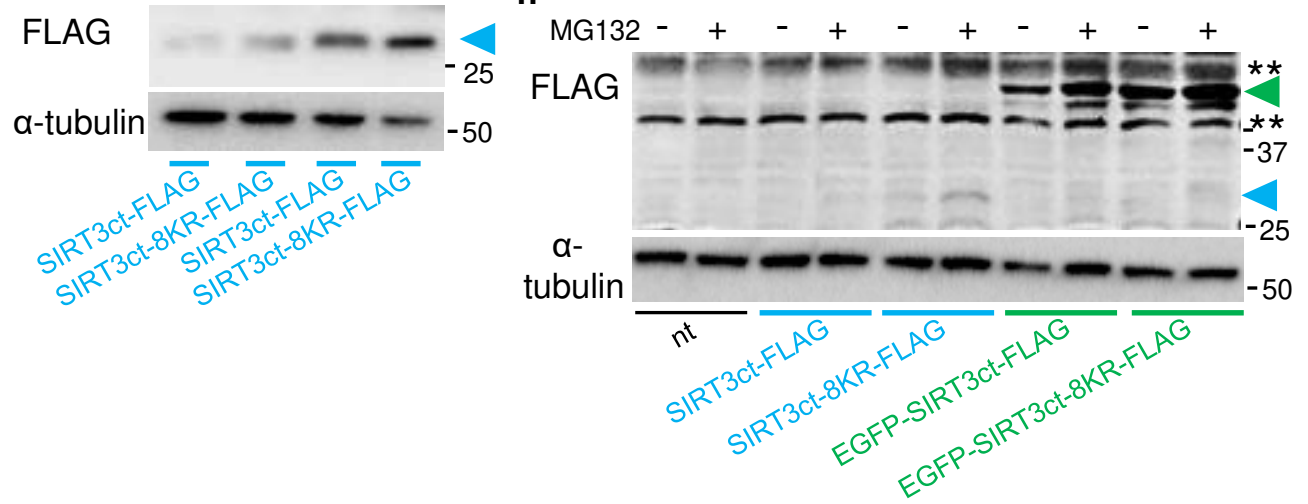
Figure 5.

a

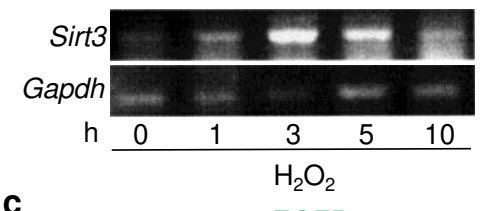

C

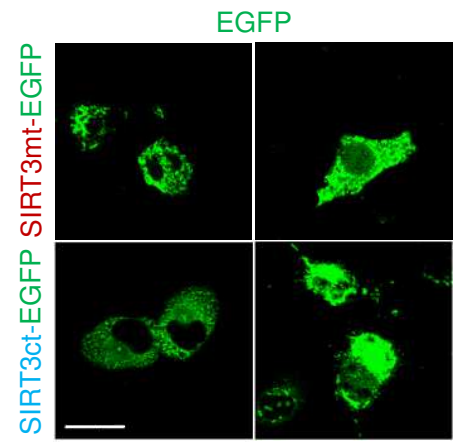

$\mathrm{H}_{2} \mathrm{O}_{2} \quad(-)$

d

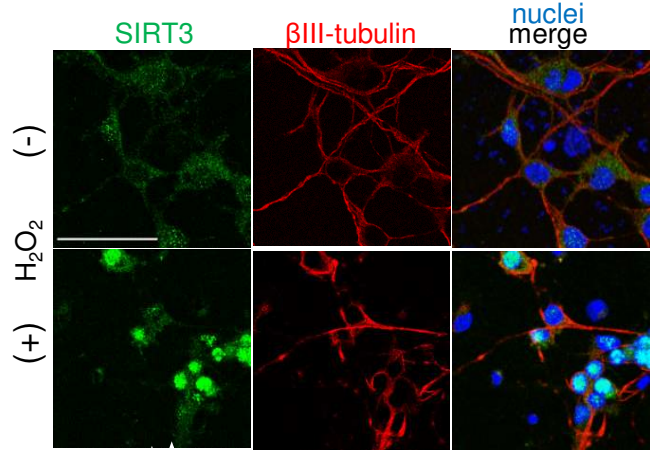

f

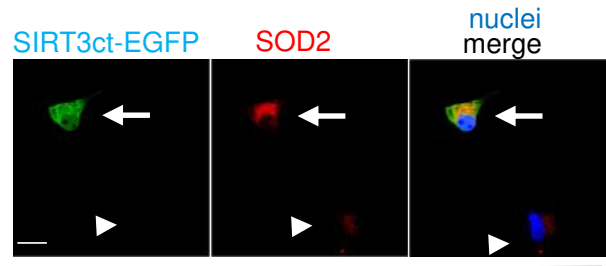

$\mathrm{H}_{2} \mathrm{O}_{2}$

g

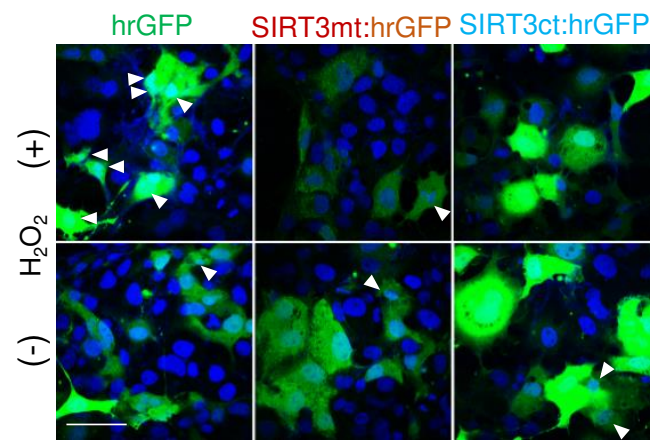

b

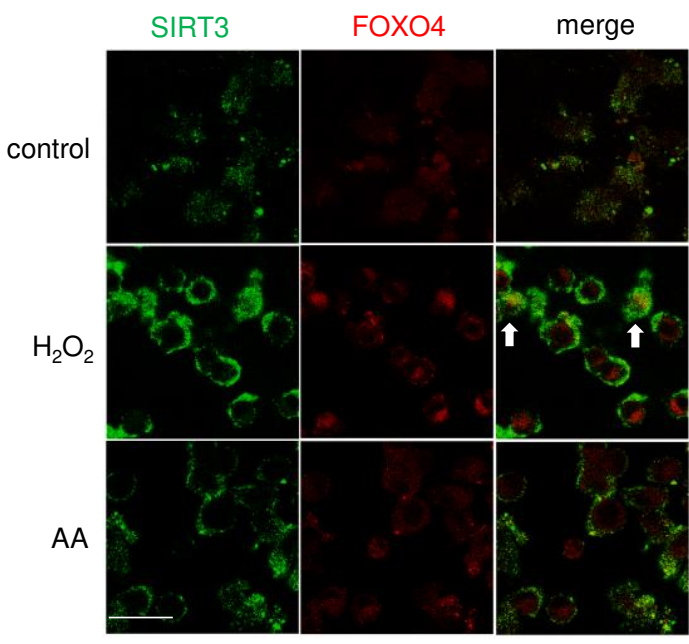

e

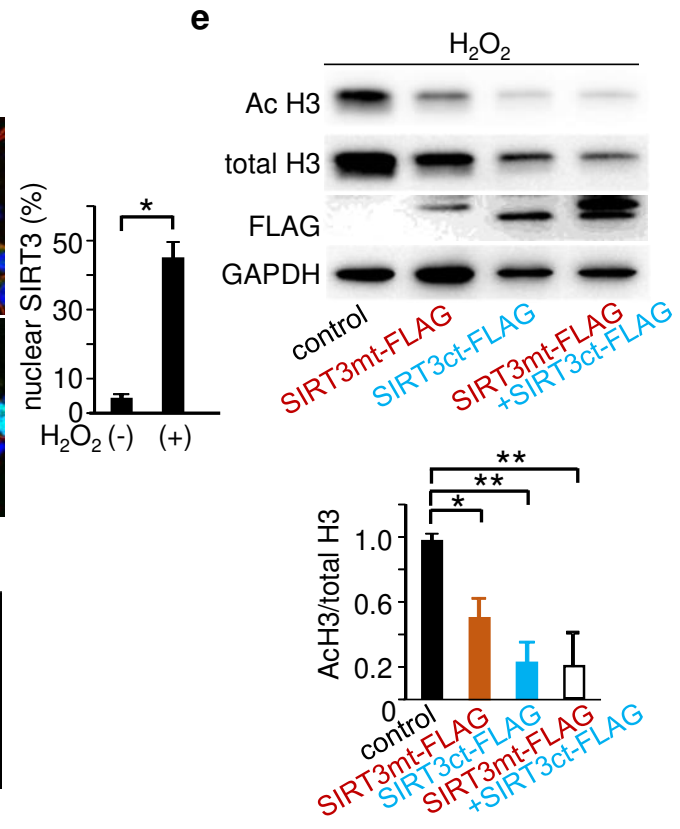

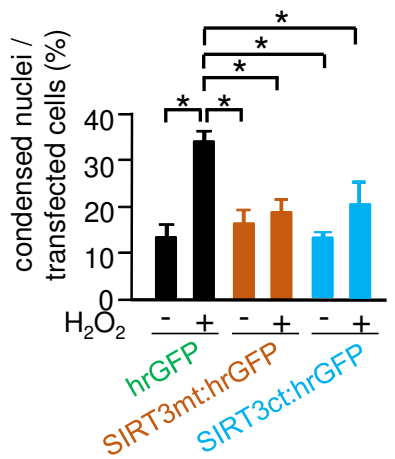


Figure 6.

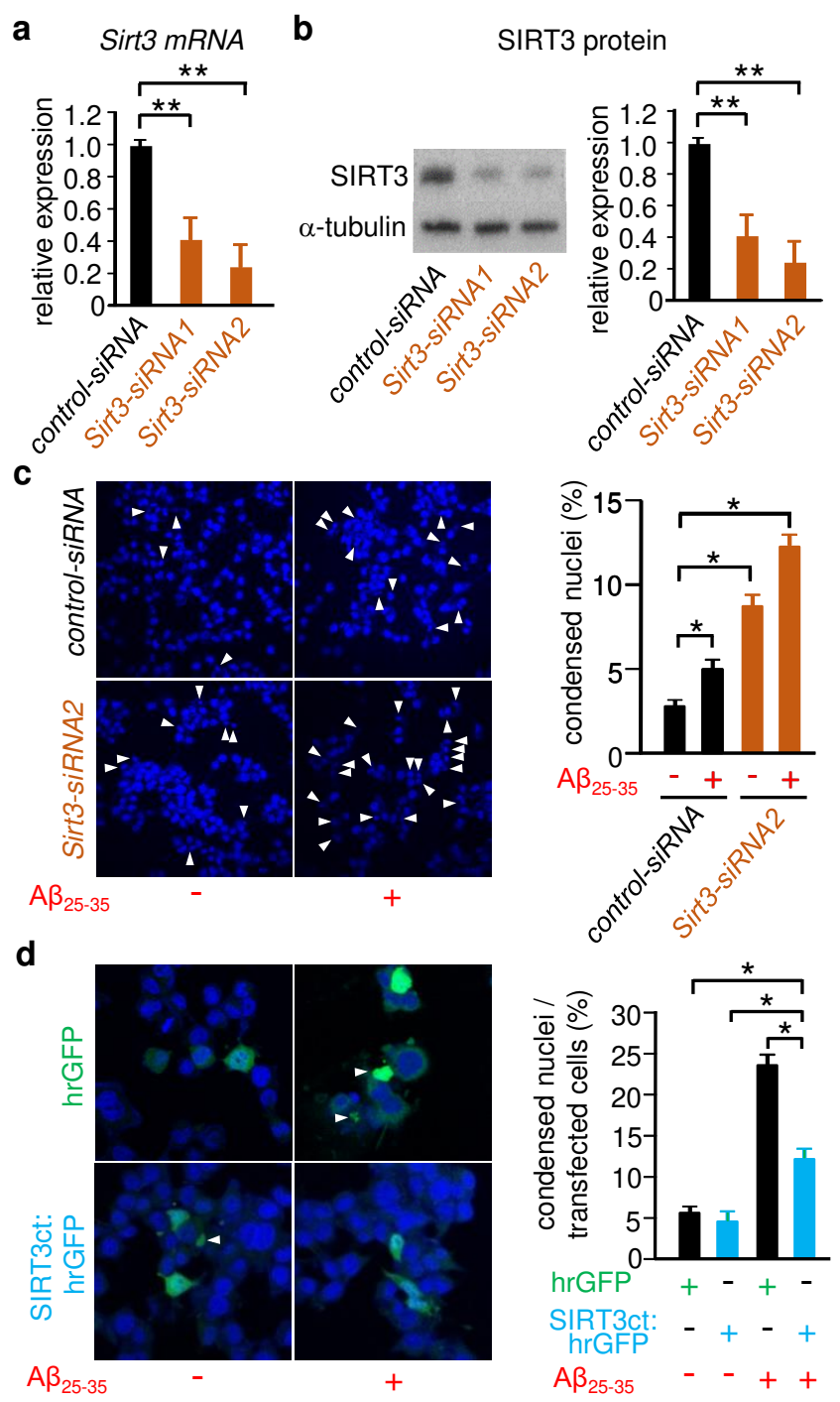




\section{Figures}

a

mouse Sirt3 gene

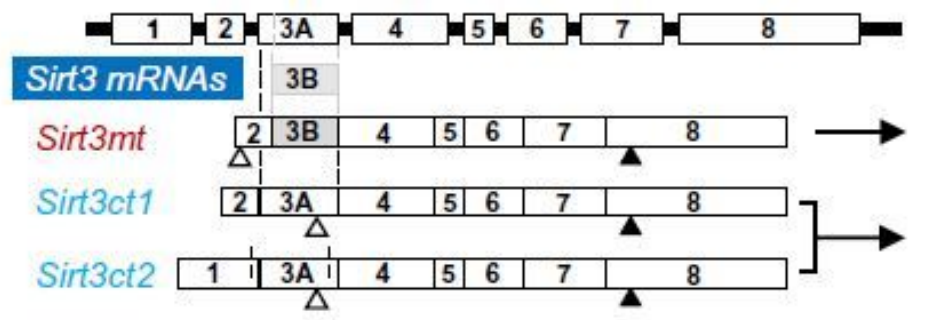

b

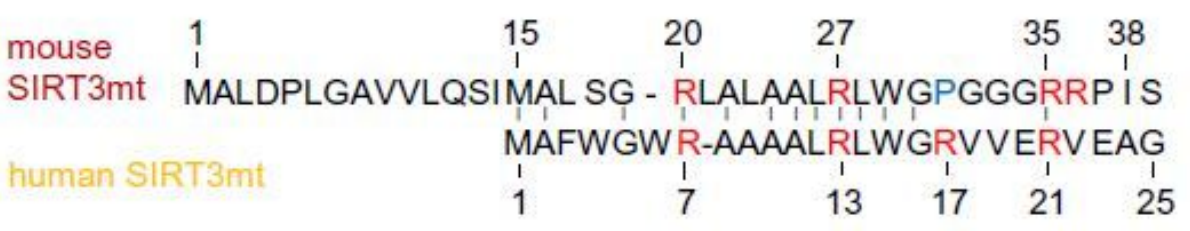

C

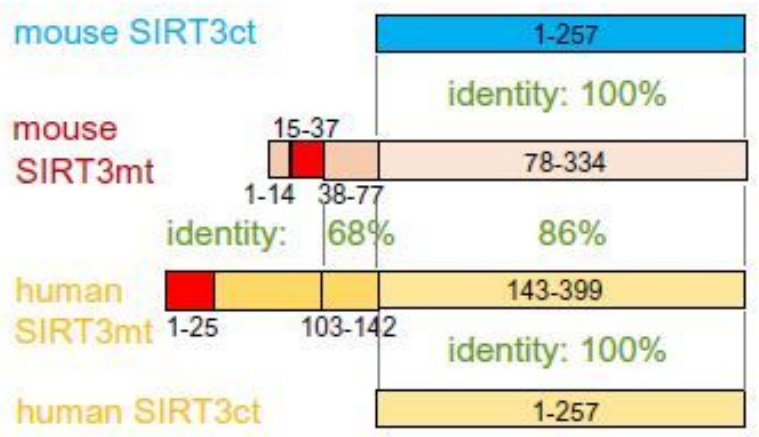

e

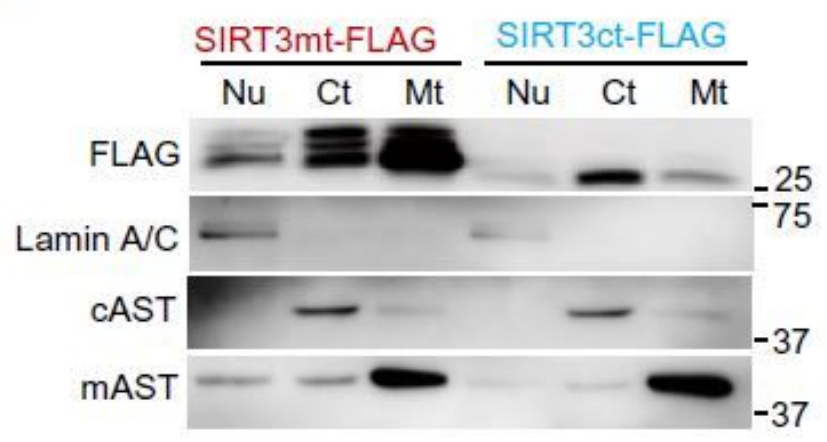

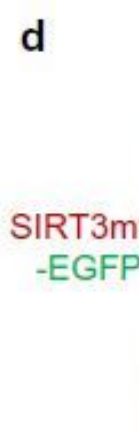

SIRT3ct -EGFP

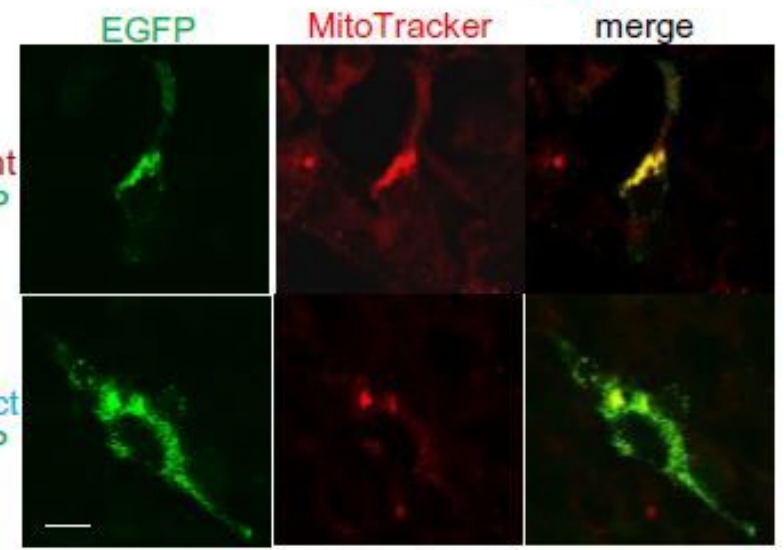

f

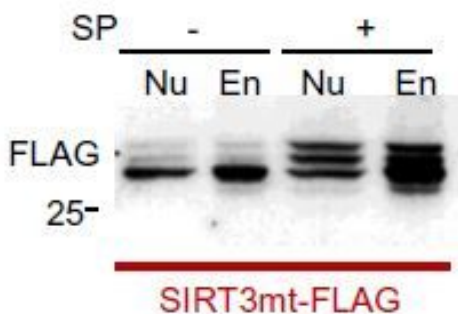

mouse SIRT3 proteins
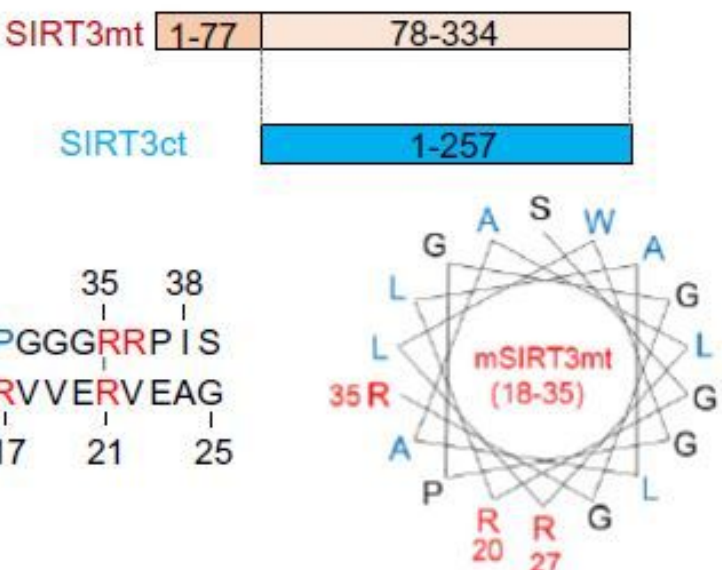

20 27 
are indicated by red and blue text, respectively (left panel). A helical wheel plot of residues 18-35 of mouse SIRT3mt shows a cluster of positively charged arginine 37 residues on one side of the helix opposed by hydrophobic amino acids on the other side, a typical feature of mitochondrial targeting sequences (right panel). Arginine residues and hydrophobic amino acid residues are indicated by red and blue text, respectively. (c) Comparison of mouse and human SIRT3ct and SIRT3mt. Identities between amino acid sequences of conserved regions are indicated with percentages (\%). Mitochondrial targeting sequence of residues 1-25 of human SIRT3mt and the corresponding region of mouse SIRT3mt are shown in red. (d) Distribution of SIRT3mt-EGFP and SIRT3ct-EGFP in COS7 cells. Mitochondria are labelled with MitoTracker Red. Scale bar: $10 \mu \mathrm{m}$. (e) COS7 cells expressing SIRT3mt-FLAG or SIRT3ctFLAG were homogenized and fractionated into nuclear $(\mathrm{Nu})$, cytoplasmic $(\mathrm{Ct})$ and mitochondrial (Mt) fractions and analysed by Western blot using an anti-FLAG antibody. The membrane filter was reblotted by antibodies of Lamin A/C, CAST and mAST, markers of nuclear, cytoplasmic and mitochondrial fractions, respectively. (f) COS7 cells expressing SIRT3mt-FLAG or SIRT3ct-FLAG were treated with a nongenotoxic stressor staurosporine $(100 \mathrm{nM})$ for $12 \mathrm{~h}$ and fractionated into nuclear $(\mathrm{Nu})$ and extra-nuclear (En) fractions. En fractions contain cytoplasm and mitochondria. 
a
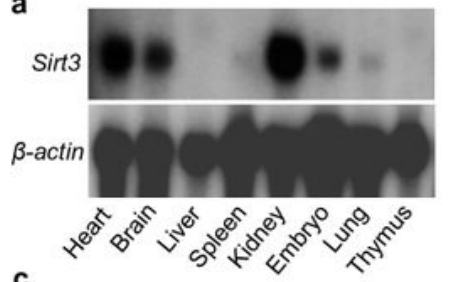

b

Sirt3

28

$18 s$

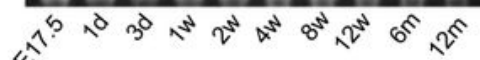

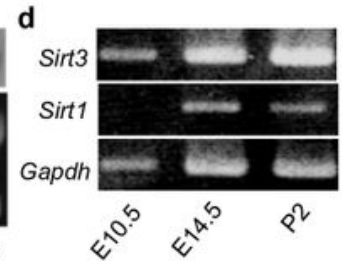

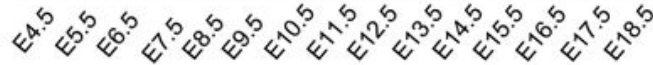

e

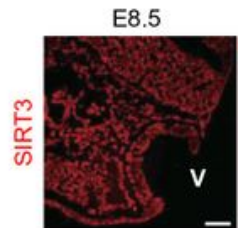

E10.5

E14.5

P2

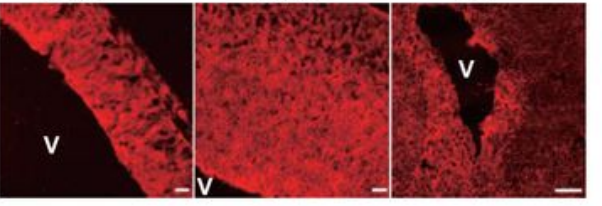

f

SIRT3

nestin

nuclei

merge

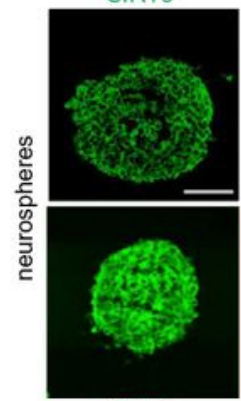

SIRT1

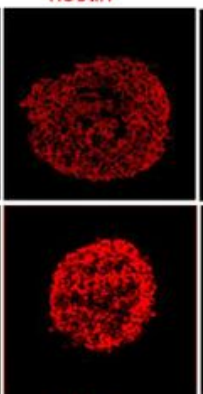

nestin

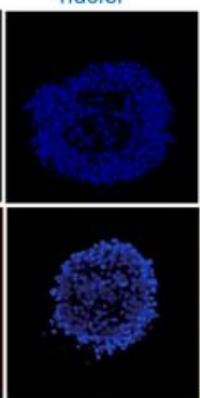

nuclei

h

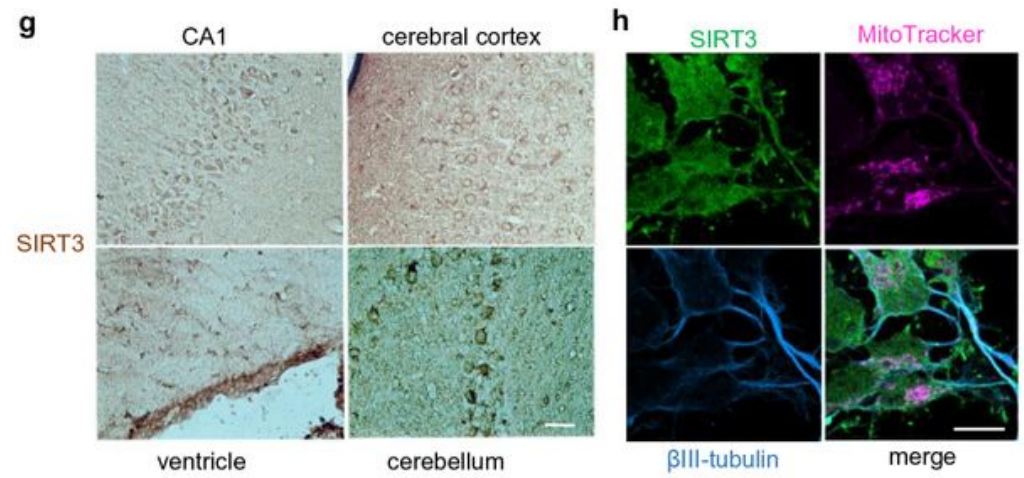

ßIII-tubulin

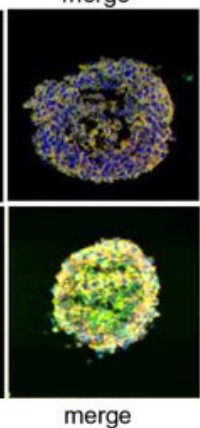

Figure 2

Expression of SIRT3 in the brain and neural precursor cells. 38 (a-c) Northern blot analyses of Sirt3 mRNA: (a) Various organs. (b) Brains of various ages. (c) Fetuses of various stages. $28 \mathrm{~s}$ and $18 \mathrm{~s}$ indicate 28 s and 18s units of ribosomal RNA, respectively. E: embryonic day, d: day, w: week, and m: month. (d) RT-PCR analysis of Sirt3 and Sirt1 in fetal and postnatal brains. (e) SIRT3 immunostaining (red) of embryonic and postnatal brains. P: postnatal day and V: ventricles. Scale bars: $50 \mu \mathrm{m}$ (E8.5), $20 \mu \mathrm{m}$ 
(E10.5 and E14.5) and $100 \mu \mathrm{m}$ (P2). (f) SIRT3 and SIRT1 immunostaining (green) of cultured neurospheres isolated from mouse embryonic brain. Nestin (red) is a marker of NPCs. Nuclei are stained with Hoechst 33342 (blue). Scale bar: $100 \mu \mathrm{m}$. (g) CA1 region of hippocampus, cerebral cortex, lateral ventricle and cerebellum were immunostained with an anti-SIRT3 antibody (brown). Scale bar: $50 \mu \mathrm{m}$. (h) SIRT3 immunostaining (green) of cultured neural cells. MitoTracker Red (purple) and Blll-tubulin (blue) are markers for mitochondria and neural cells, respectively. Scale bar: $10 \mu \mathrm{m}$.

a

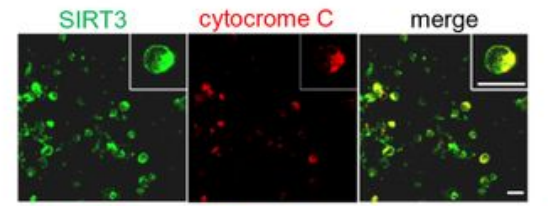

b

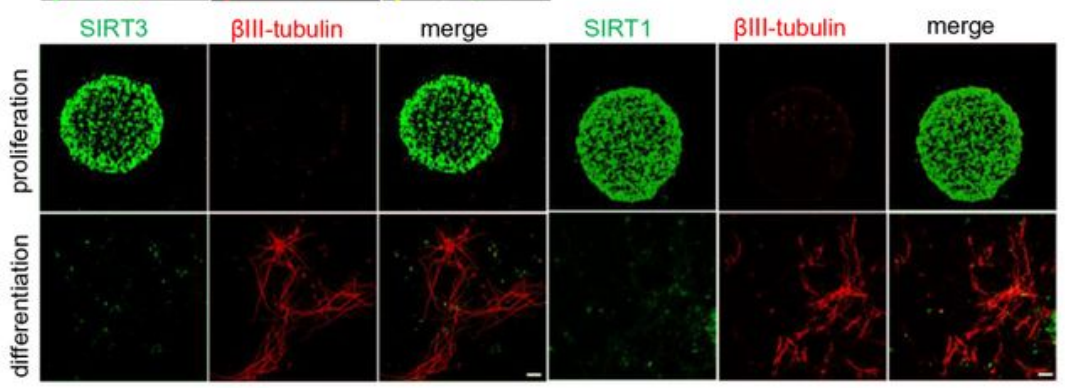

c

d
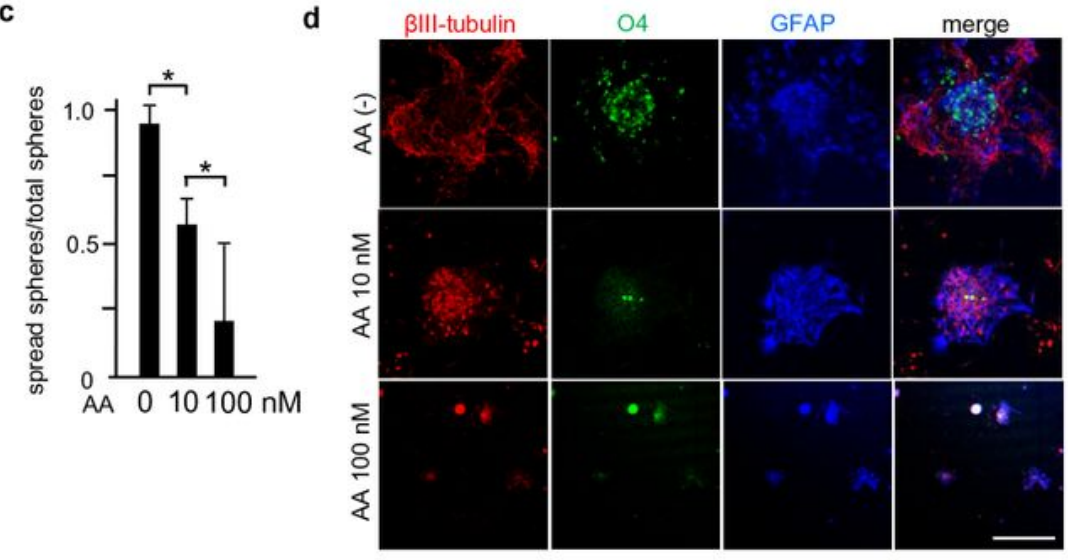

BIII-tubulin

GFAP

merge

e

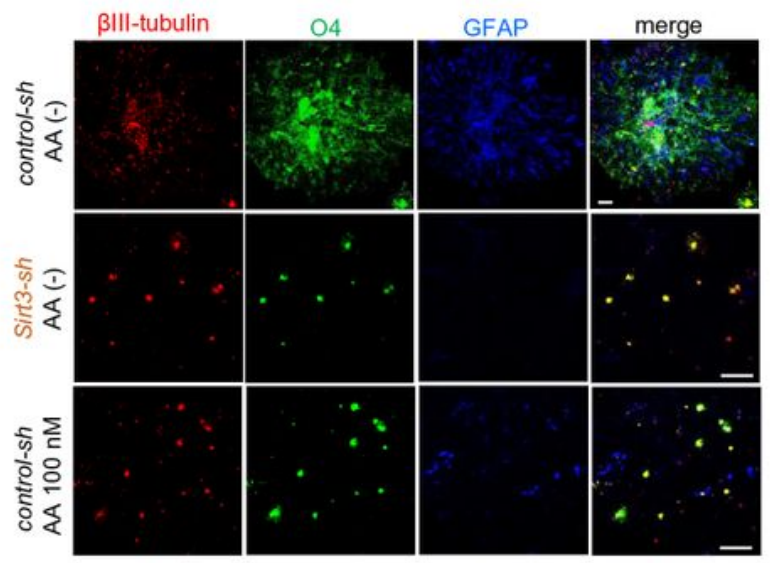

Figure 3 
SIRT3 knockdown disturbed neural precursor cell differentiation. (a) NPCs mechanically dissociated from cultured neurospheres were spread on poly-L-ornithine-coated glasses and immunostained with an antiSIRT3 (green) and an anti-cytochrome C (a marker of mitochondria, red) antibodies. Scale bar: $10 \mu \mathrm{m}$. (b) Expression of SIRT3 (green), SIRT1 (green) and $\beta$ III-tubulin (red) of undifferentiated 39 neurospheres under proliferation conditions and differentiated neurospheres. Scale bar: $50 \mu \mathrm{m}$. (c) Neurospheres were differentiated in the presence or absence of AA for 5 days. The number of neurospheres with dissociated cells as well as round undifferentiated neurospheres were counted and compared. ${ }^{*}<0.01$. (d) Neurospheres were differentiated with a differentiation medium in the presence or absence of AA for 5 days and immunostained with ßlll-tubulin (red), 04 (an oligodendrocyte marker, green) and GFAP (an astrocyte marker, blue) antibodies. Scale bar: $50 \mu \mathrm{m}$. (e) Neurospheres were infected with lentiviruses expressing control-shRNA or Sirt3-shRNA. One day after the infection, cells were differentiated in the differentiation medium for 5 days and then immunostained. In some experiments AA was added into the differentiation medium. Scale bars: $50 \mu \mathrm{m}$. 
a

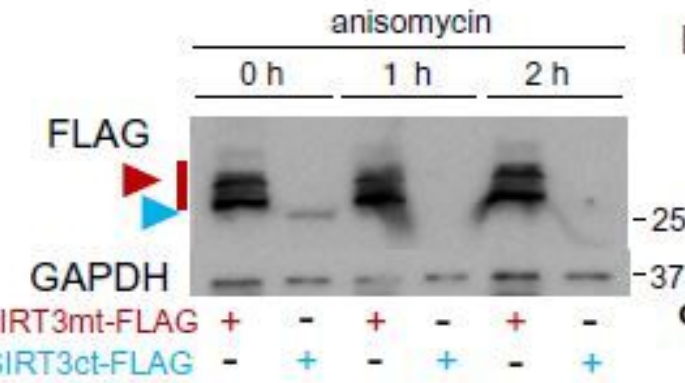

b

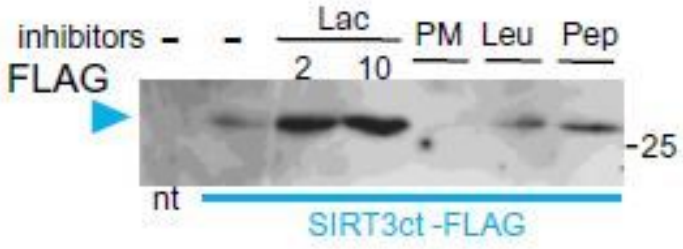

d SIRT3ct

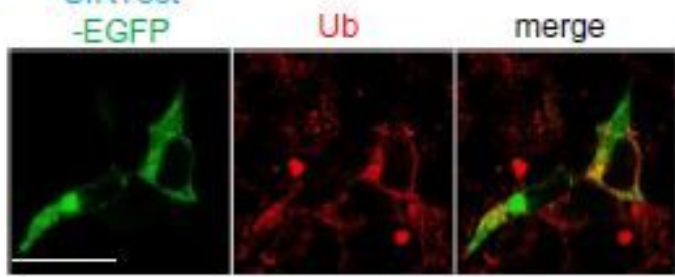

g

e
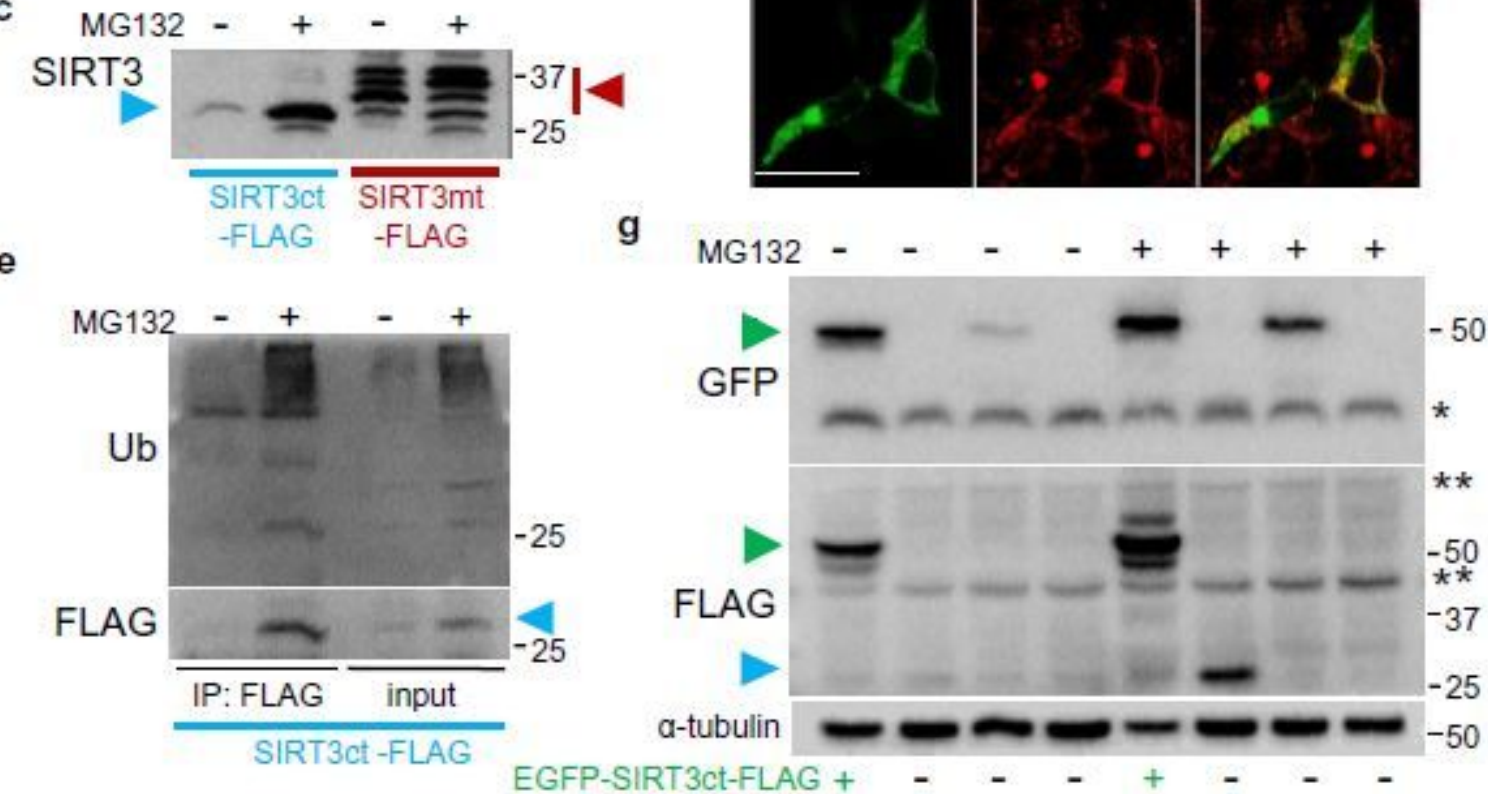

f

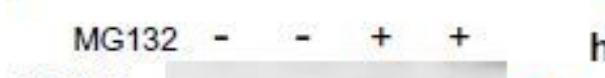

SIRT3Ct-FLAG - $+-\overline{-}+\overline{-}=$

SIRT3ct-EGFP - - + - --+

FLAG

h

no transfection - --+---+

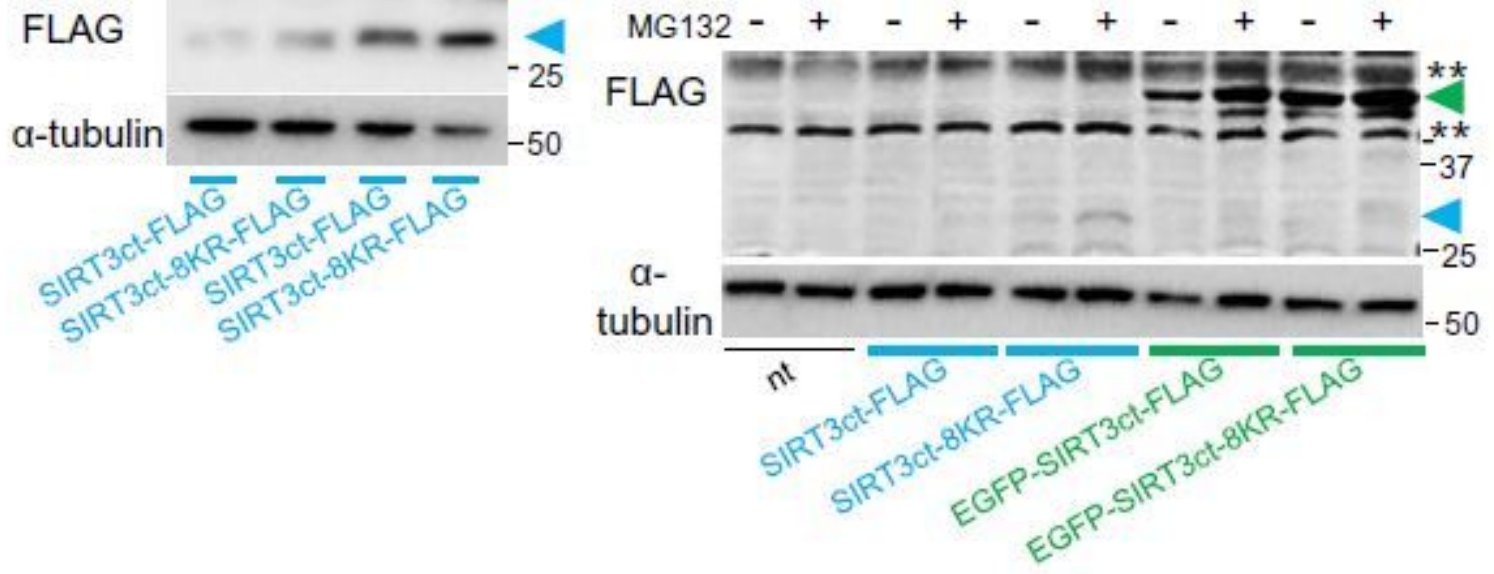

\section{Figure 4}

Ubiquitin-dependent degradation of SIRT3ct protein. (a) COS7 cells transfected with SIRT3mt-FLAG or SIRT3ct-FLAG cDNA were treated with $10 \mu \mathrm{M}$ anisomycin for the indicated times and analysed by Western blot. The filter was probed with an anti-FLAG antibody and then rebotted with an anti-GAPDH antibody. (b) COS7 cells transfected with SIRT3ct-FLAG cDNA were treated with various protease inhibitors for $10 \mathrm{~h}$ and analysed with an anti-FLAG antibody. Lac: $2 \mu \mathrm{M}$ or $10 \mu \mathrm{M}$ lactacystin, PM: $1 \mathrm{mM}$ 
PMSF, Leu: $100 \mu \mathrm{M}$ leupeptin and Pep: $100 \mu \mathrm{M} 40$ pepstatin. (c) HEK293T cells transfected with SIRT3mtFLAG or SIRT3ct-FLAG cDNA were treated with $100 \mu \mathrm{M} \mathrm{MG132}$ for $10 \mathrm{~h}$ and analysed with an anti-SIRT3 antibody. (d) Immunostaining of ubiquitin (Ub, red) in COS7 cells transfected with SIRT3ct-EGFP cDNA. Scale bar: $20 \mu \mathrm{m}$. (e) COS7 cells transfected with SIRT3ct-FLAG cDNA were incubated in the presence or absence of $100 \mu \mathrm{M} \mathrm{MG132}$ for $10 \mathrm{~h}$, homogenized, immunoprecipitated with anti-FLAG antibody-resin, and then analysed with an anti-ubiquitin antibody. The filter was reblotted with an anti-FLAG antibody. (f) COS7 cells transfected with SIRT3ct-FLAG or SIRT3ct-8KR-FLAG cDNA were incubated for $48 \mathrm{~h}$ and then treated with MG132 for $10 \mathrm{~h}$. Cells were homogenized and analysed by Western blot. (g) COS7 cells transfected with EGFP-SIRT3ct-FLAG, SIRT3ct-FLAG, or SIRT3ct-EGFP cDNA were incubated for $48 \mathrm{~h}$ and then treated with or without MG132 for $10 \mathrm{~h}$. Cells were analysed by an anti-GFP antibody and then sequentially reblotted with an anti-FLAG and an anti-GAPDH antibodies. Green and blue arrowheads indicate EGFP-fused SIRT3ct and FLAG-tagged SIRT3ct proteins, respectively. * and ** indicate nonspecific bands by the an anti-GFP antibody and an anti-FLAG antibody, respectively. (h) COS7 cells transfected with SIRT3ct-FLAG, SIRT3ct-8KR-FLAG, EGFP-SIRT3ct-FLAG, or EGFP-SIRT3ct-8KR-FLAG CDNA 41 were incubated for $48 \mathrm{~h}$ and treated with or without MG132 for $10 \mathrm{~h}$. Cells were homogenized and analysed by Western blot. nt: no transfection. 
a

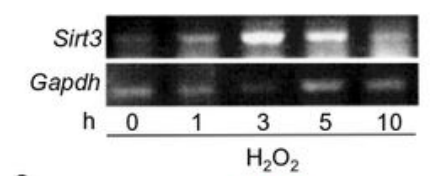

c

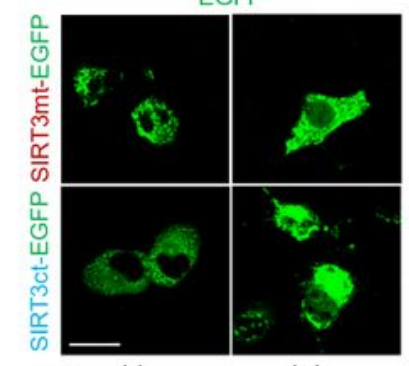

(+)

b
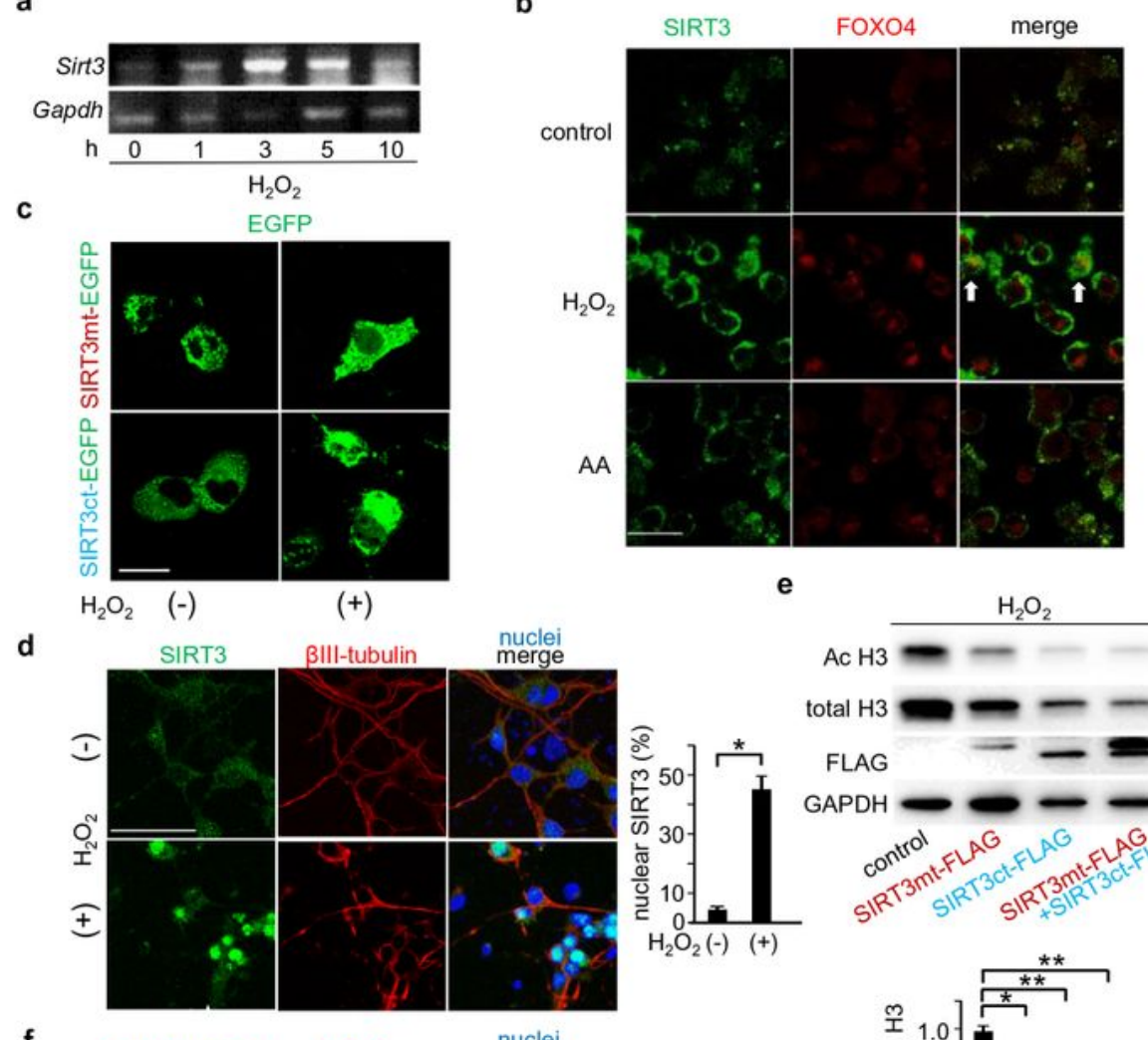

f
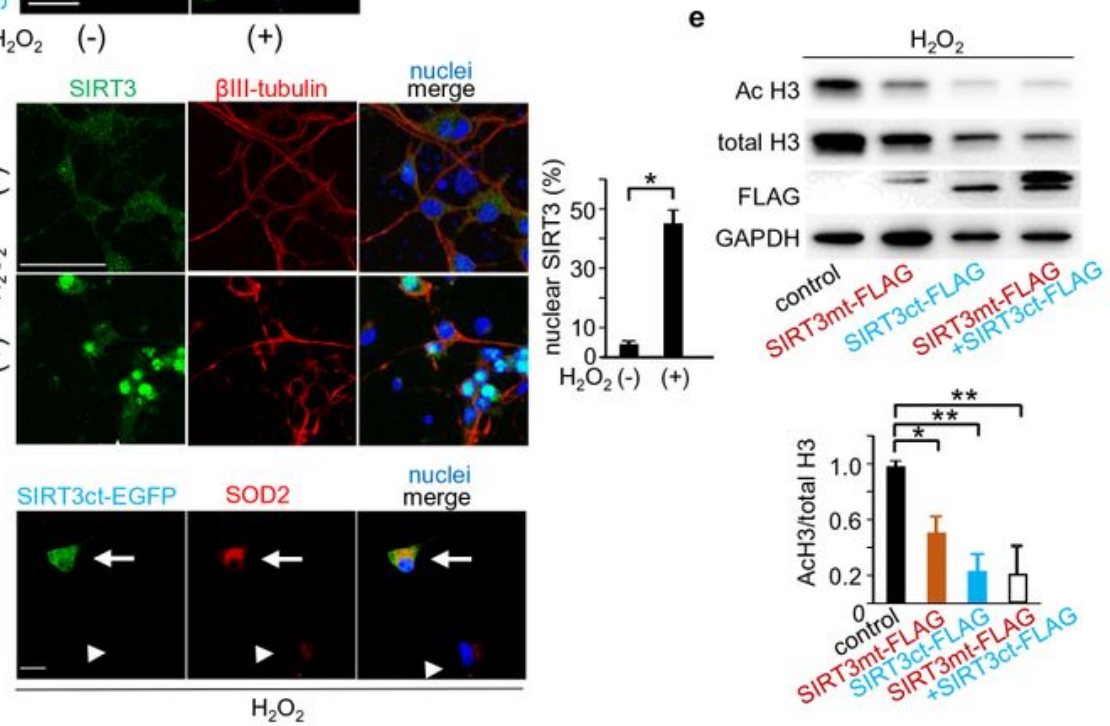

g
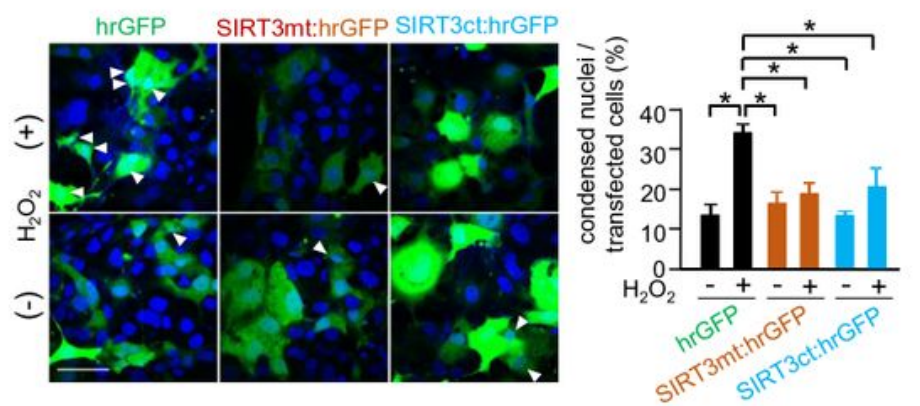

\section{Figure 5}

Nuclear translocation of SIRT3ct protected cells against oxidative stress. (a) PC12 cells were treated with $200 \mu \mathrm{M} \mathrm{H} 2 \mathrm{O} 2$ for the indicated hours and analysed by RT-PCR. (b) PC12 cells were treated with $100 \mu \mathrm{M}$ $\mathrm{H} 2 \mathrm{O} 2$ or $100 \mathrm{nM}$ antimycin $\mathrm{A}(\mathrm{AA})$ for $12 \mathrm{~h}$ and then analysed by immunostaining of SIRT3 (green) and FOXO4 (red). Arrows indicate nuclear staining of SIRT3 and FOXO4. Bar: $20 \mu \mathrm{m}$. (c) COS7 cells transfected with SIRT3mt-EGFP or SIRT3ct-EGFP cDNA were treated with or without $100 \mu \mathrm{M} \mathrm{H} 2 \mathrm{O} 2$ for 12 
h. Bar: $20 \mu \mathrm{m}$. (d) Mouse primary cortical neurons were treated with $500 \mathrm{nM} \mathrm{H} 2 \mathrm{O} 2$ for $12 \mathrm{~h}$ and stained

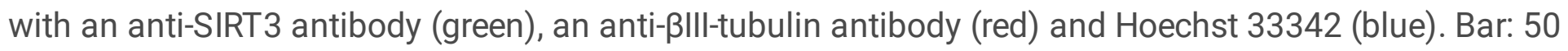
$\mu \mathrm{m}$. The numbers of cells expressing cytoplasmic SIRT3 and nuclear SIRT3 were counted and compared (right panel). ${ }^{*} p<0.001$. (e) COS7 cells transfected with SIRT3mt-FLAG, SIRT3ct-FLAG cDNA or both plasmids were treated with $100 \mu \mathrm{M} \mathrm{H} 2 \mathrm{O} 2$ and $25 \mathrm{nM}$ trichostatin A, an inhibitor of NAD+-independent histone deacetylases, for $12 \mathrm{~h}$. The expression levels of acetylated histone $\mathrm{H} 3$ and total histone $\mathrm{H} 3$ were analysed by Western blot. The filter blotted with an anti-acetyl histone $\mathrm{H} 3$ and an anti-GAPDH antibodies was sequentially reblotted with an anti-histone $\mathrm{H} 3$ antibody and an anti-FLAG antibody. Intensities of bands were 42 compared $(n=3) .{ }^{*} p<0.05, * \star p<0.01$. (f) PC12 cells transfected with SIRT3ct-EGFP cDNA were incubated for $48 \mathrm{~h}$ and then treated with $100 \mu \mathrm{M} \mathrm{H} 2 \mathrm{O} 2$ for $12 \mathrm{~h}$. The cells were immunostained with an anti-SOD2 antibody (red). Nuclei were stained with Hoechst 33342. The arrow and arrowhead indicate SIRT3ct-EGFP+ cells and SIRT3ct-EGFP- cells, respectively. Bar: $20 \mu \mathrm{m}$. (g) COS7 cells transfected with the pIRES-hrGFP vector, SIRT3mt:hrGFP or SIRT3ct:hrGFP plasmid, were incubated for $24 \mathrm{~h}$ and then treated with $100 \mu \mathrm{M} \mathrm{H} 2 \mathrm{O} 2$ for $24 \mathrm{~h}$. Cells were then stained with Hoechst 33342 (blue). The numbers of hrGFP+ cells with condensed nuclei and those of hrGFP+ cells without condensed nuclei in a visual field were counted. Five visual fields were counted in each condition. The percentages of dead hrGFP+ cells in total hrGFP+ cells are shown (right panel). ${ }^{*} p<0.05$. Scale bar: $20 \mathrm{Dm}$. 


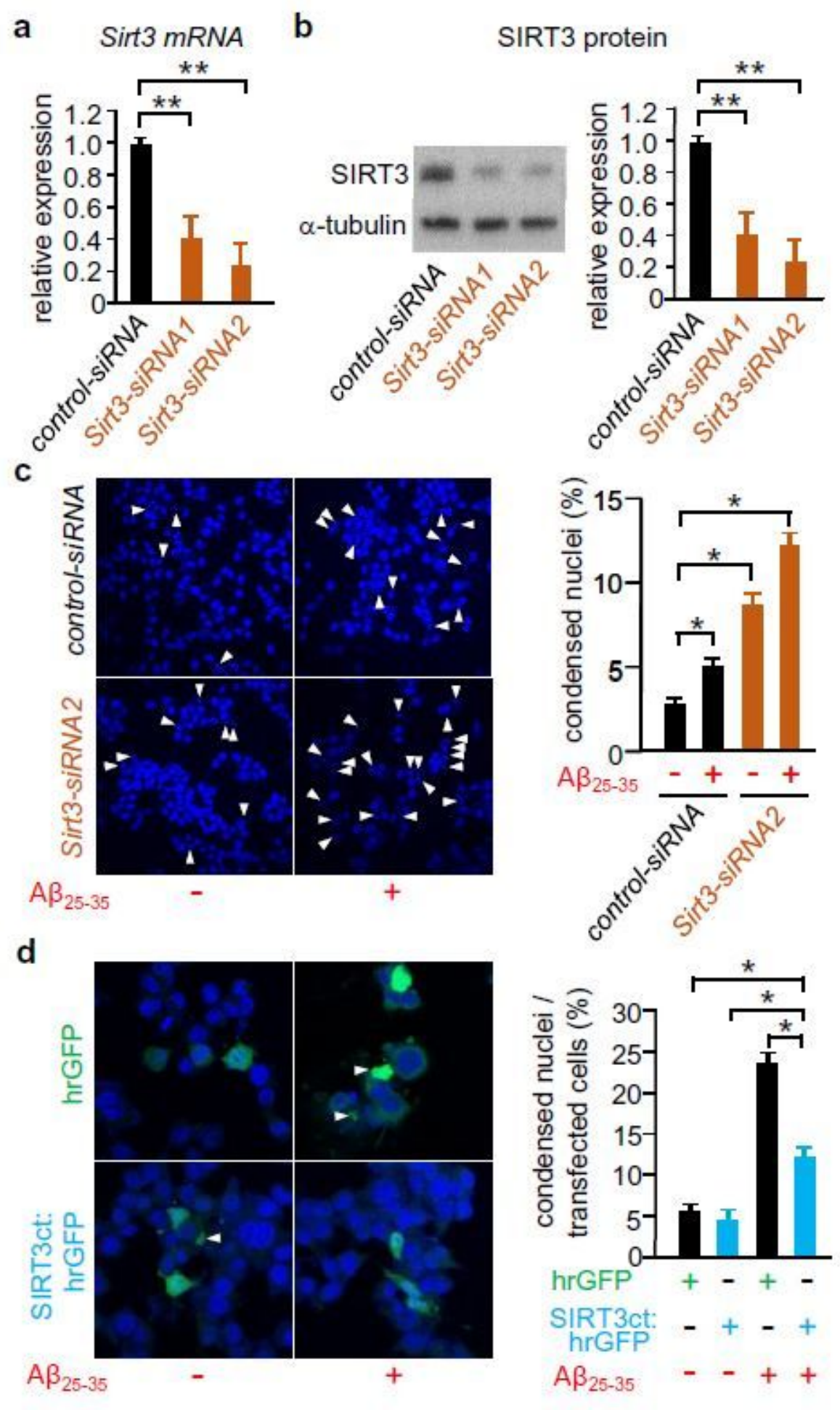

\section{Figure 6}

SIRT3ct protected cells against A $25-35$ toxicity. $(a, b)$ PC12 cells were transfected with control-siRNA, Sirt3-siRNA1 or Sirt3-siRNA2. Expression levels of Sirt3 mRNA (a) and SIRT3 protein (b) were compared after $48 \mathrm{~h}$ of transfection. ${ }^{* *} p<0.01$. (c) PC 12 cells were transfected with control-siRNA or Sirt3-siRNA2 and then treated with $50 \mu \mathrm{M}$ Aß25-35 $24 \mathrm{~h}$ after transfection. Cells were further incubated for an additional $24 \mathrm{~h}$ and then stained with Hoechst 33342 to detect dead cells. Condensed apoptotic nuclei 
are indicated by arrowheads. (d) PC12 cells transfected with pIRES-43 hrGFP or SIRT3ct:hrGFP plasmid were incubated for $24 \mathrm{~h}$ and then treated with $50 \mu \mathrm{M}$ A $325-35$ for $24 \mathrm{~h}$. Cells were stained with Hoechst 33342 to detect apoptotic cells. Number of apoptotic hrGFP+ cells were compared with those of hrGFP+ cells. ${ }^{*} p<0.05,{ }^{* *} p<0.01$.

\section{Supplementary Files}

This is a list of supplementary files associated with this preprint. Click to download.

- 201201SupplementaryfilebyHayashietal..pdf 